\title{
AN APPROXIMATION SOLVABILITY METHOD FOR NONLOCAL DIFFERENTIAL PROBLEMS IN HILBERT SPACES
}

\author{
Irene Benedetti \\ Department of Mathematics and Informatics \\ University of Perugia, Perugia, Italy \\ irene.benedetti@dmi.unipg.it \\ Nguyen Van Loi \\ Faculty of Fundamental Science \\ PetroVietNam University, Ba Ria - Vung Tau, Vietnam \\ loinv@pvu.edu.vn \\ Luisa Malaguti \\ Department of Sciences and Methods for Engineering \\ University of Modena and Reggio Emilia, Reggio Emilia, Italy \\ luisa.malaguti@unimore.it \\ Valeri Obukhovskii \\ Faculty of Physics and Mathematics \\ Voronezh State Pedagogical University, Voronezh, Russia \\ valerio-ob2000@mail.ru
}

Received 19 September 2014

Revised 30 September 2015

\begin{abstract}
A new approach is developed for the solvability of nonlocal problems in Hilbert spaces associated to nonlinear differential equations. It is based on a joint combination of the degree theory with the approximation solvability method and the bounding functions technique. No compactness or condensivity condition on the nonlinearities is assumed. Some applications of the abstract result to the study of nonlocal problems for integrodifferential equations and systems of integro-differential equations are then showed. A generalization of the result by using nonsmooth bounding functions is given.
\end{abstract}

Keywords: Nonlocal condition; differential equation; degree theory; approximation solvability method; bounding function; integro-differential equation.

Mathematics Subject Classification 2010: Primary: 34B10. Secondary: 34A34, 35R09, $47 \mathrm{H} 11$ 
2 Benedetti, Loi, Malaguti and Obukhovskii

\section{Introduction}

Let $H$ be a separable Hilbert space. In this paper we consider a differential equation of the form:

$$
x^{\prime}(t)=f(t, x(t)) \text { for a.e. } t \in[0, T]
$$

with a nonlocal condition

$$
x(0)=M x,
$$

where $f:[0, T] \times H \rightarrow H$ and $M: C([0, T] ; H) \rightarrow H$ are nonlinear and linear maps, respectively.

It is well known (see, e.g., [?,?] and the references therein) that a great variety of partial differential and integro-differential equations can be written in the form (??).

In this paper, by combining the degree theory in abstract spaces with an improvement of the approximation solvability method and the bounding functions technique an existence theorem (see Theorem ??) for problem (??)-(??) is proved. It is then showed how the abstract result can be applied to study various problems of integro-differential equations (including periodic, anti-periodic, mean value and multi-point problems).

Compactness conditions in terms of the strong topology are usually required in order to apply the degree theory for a suitable solution operator. In this paper we obtain existence results in the lack of this compactness both on the nonlinear term $f$ and on the nonlocal operator $M$. This is possible exploiting compactly embedded Gel'fand triples with a Hilbert space and Hartman-type conditions. Other techniques have previously been employed to avoid assumptions of compactness. For instance in [?] a continuation principle has been used in the weak topology in reflexive Banach spaces and in [?] the concept of weak measure of non compactness is introduced to consider also the case of non reflexive Banach spaces.

The paper is organized in the following way. In the next section we give a brief review of all the methods which will be used to study problem (??)-(??). We explain also the main idea of our technique presented in this paper. Section 3 is devoted to the Leray-Schauder topological degree and the notation. The main result is presented in Section 4. Some applications of the abstract result are given in Section 5 including the periodic problem for an integro-differential equation of the form

$$
\left\{\begin{array}{l}
u_{t}+a \int_{\Omega} u(t, \xi) d \xi=-b u(t, \xi)+f(t, u(t, \xi)), \\
u(0, \xi)=u(1, \xi)
\end{array}\right.
$$

for a.e. $t \in[0,1]$ and for all $\xi \in \Omega \subset \mathbb{R}^{n}(n \geq 2)$, where $a, b>0$ and $f:[0,1] \times \mathbb{R} \rightarrow \mathbb{R}$ is a continuous function; and a mean value problem of the form

$$
\left\{\begin{array}{l}
u_{t}+a u(t, s) d s=\int_{0}^{1} k(s, \xi)(u(t, \xi)+b) d \xi \\
u(0, s)=\int_{0}^{1} u(t, s)
\end{array}\right.
$$


for a.e. $t \in[0,1]$ and for all $s \in[0,1]$, where $a>0 ; b \in \mathbb{R}$ and $k:[0,1] \times[0,1] \rightarrow \mathbb{R}$ is a smooth function.

In Section 6, equation (??) is considered for $t \in[0, \infty)$ and with the initial condition $x(0)=0$. The existence of a solution which is bounded on the whole interval $[0, \infty)$ is showed. In Section 7 we deal with systems of differential equations in various Hilbert spaces with application to a system of integro-differential equations with non-local conditions

$$
\left\{\begin{array}{l}
\frac{\partial u(t, \xi)}{\partial t}+a \int_{\Omega} u(t, \xi) d \xi+b u(t, \xi)=f_{1}(t, u(t, \xi), v(t, \xi)), \\
\frac{\partial v(t, \xi)}{\partial t}+c v(t, \xi)=f_{2}(t, u(t, \xi), v(t, \xi)) \\
u(0, \xi)=\sum_{1}^{m} \alpha_{j} u\left(t_{j}, \xi\right) \\
v(0, \xi)=\int_{0}^{1} g(t) v(t, \xi) d t
\end{array}\right.
$$

for a.e. $t \in[0,1]$ and for all $\xi \in \Omega \subset \mathbb{R}^{n}(n \geq 2)$, where $a, b, c>0 ; \alpha_{j} \in \mathbb{R}$; $0<t_{1}<\cdots<t_{m} \leq 1 ; g \in L^{1}[0,1] ;$ and $f_{i}:[0,1] \times \mathbb{R} \times \mathbb{R} \rightarrow \mathbb{R}(i=1,2)$ are continuous maps.

In Section 8 we investigate the existence of a unique solution for all the problems considered in the paper. In the last section the generalization of the main result is presented by using a nonsmooth bounding function.

\section{A review of methods}

The technique presented in this paper is based on the bounding functions method and the approximation method. So, let us give a brief review of both of them.

Bounding functions method: The earliest version of a bounding function is a guiding function which was introduced by Krasnosel'skii and Perov (see, e.g., [?,?, ?]). They generalized the notion of the Lyapunov function to study the existence of periodic solutions of an ODE

$$
x^{\prime}(t)=f(t, x(t)),
$$

where $f: \mathbb{R} \times \mathbb{R}^{n} \rightarrow \mathbb{R}^{n}$ is a (globally) continuous map which is locally Lipschitzian w.r.t. the second argument. The notion of guiding function was then generalized in several directions and applied to various problems. Among a large number of papers on this subject let us recall: Mawhin [?] with studying of functional differential equations; Fonda [?] with the notion of integral guiding function; Capietto and Zanolin [?] for periodic problem in flow-invariant Euclidean Neighborhood Retracts; Górniewicz and Plaskacz [?,?] with the notion of general form of guiding functions for differential inclusions (see also [?]); Lewicka [?] for nonsmooth guiding functions; Kryszewski [?], Kryszewski and Gabor [?] and Loi [?] with the application of the method of guiding functions to bifurcation problems. The backgrounds and applications of the method of guiding functions in nonlinear analysis can be found in the recent monograph [?].

However, it seems to be that the method of guiding functions can hardly be applied to study more general classes of boundary value problems than periodic 
problem. Also it is worth noting that usually the direct applications of the method of guiding functions were connected with finite-dimensional objects.

In [?] (see also [?]) Mawhin introduced the concept of bounding functions, this method was then developed by Gaines and Mawhin [?,?] and by Mawin and Ward [?].

We recall in the following the main features of this method. Consider again equation (??) for $t \in[a, b]$ with the boundary condition

$$
g(x(a), x(b))=0,
$$

where $f$ and $g$ are continuous maps. The idea for the existence of solutions to (??)(??) comes from the Leray-Schauder continuation principle [?], following which it is possible to consider the linearized problem

$$
x^{\prime}(t)=\lambda f(t, y(t)), \quad t \in(a, b),
$$

with boundary condition (??), where $\lambda \in[0,1]$ and $y \in C\left([a, b] ; \mathbb{R}^{n}\right)$ is a given function. It is supposed that for each $y(\cdot)$ problem (??)-(??) has a unique solution $T(y, \lambda)$ and moreover, the solution map

$$
T: C\left([a, b] ; \mathbb{R}^{n}\right) \times[0,1] \rightarrow C\left([a, b] ; \mathbb{R}^{n}\right),
$$

is completely continuous. Notice that a fixed point of the map $T(\cdot, 1)$ is a solution of problem (??)-(??). Now, if there exists an open bounded subset $\Omega \subset C\left([a, b] ; \mathbb{R}^{n}\right)$ such that:

(a) $\operatorname{deg}(i-T(\cdot, 0), \bar{\Omega}) \neq 0$;

(b) $x \neq T(x, \lambda)$ for all $(x, \lambda) \in \partial \Omega \times(0,1)$,

then problem (??)-(??) admits a solution $x \in \bar{\Omega}$.

Recall (see [?]) that a $C^{1}$-function $V: \mathbb{R}^{n} \rightarrow \mathbb{R}$ is said to be a bounding function to equation (??) if

(i) the set $K=\left\{x \in \mathbb{R}^{n}: V(x)<0\right\}$ is bounded and $V_{\text {loK }}=0$;

(ii) $\langle\operatorname{grad} V(x), f(t, x)\rangle \neq 0$ for all $t \in(a, b)$ and $x \in \partial K$.

The name bounding function comes from the fact that if there exists a bounding function $V$ and if there exists a fixed point $x$ of $T(\cdot, \lambda), \lambda \in(0,1)$, such that $x(a) \notin \partial K$ and $x(b) \notin \partial K$, then $x(t) \in K$ for all $t \in[a, b]$ (see [?]). Therefore, the set $\Omega$ can be taken as the set of all continuous functions $x:[a, b] \rightarrow K$. The conditions $x(a) \notin \partial K$ and $x(b) \notin \partial K$ usually follow from the choice of the boundary map $g$ (see, [?] and the last section of the present paper). To obtain condition (a) in various papers (and in the present one) the authors usually consider a convex set $K$ containing 0 . Then the linearized problem is modified so that 0 should be the unique fixed point of $T(\cdot, 0)$, and therefore, $\operatorname{deg}(i-T(\cdot, 0), \bar{\Omega})=1$.

From the above consideration it is clear that the bounding functions method is useful to study boundary value problems. Moreover, since by applying this method we do not need to evaluate the topological degree of bounding functions, it is possible 
to extend this method to infinite-dimensional Banach spaces.

Preliminary ideas of the bounding functions methods in the framework of periodic solutions were introduced in Lefschetz [?] for second order equations in $\mathbb{R}$ and in Browder [?] for dynamics in Hilbert spaces with monotone nonlinearities w.r.t. the state variable. Concerning the most important developments of this method: Mawhin and Thompson [?] for the introduction of Hartman-type conditions (see (ii) above) which are strictly located on $\partial K$; Taddei [?] for nonsmooth bounding functions in finite-dimensional spaces; Loi and Obukhovskii [?] for application of this method to generalized periodic problem in finite-dimensional spaces; Zanolin [?] for generalized definition of bound set; Loi, Kornev, Obukhovskii and Zecca for the extensions of this method to equations and inclusions in Hilbert spaces (see, $[?, ?, ?]) ;$ Andres, Malaguti and Taddei [?] for bounding functions in Banach spaces; Benedetti, Malaguti and Taddei [?] for bounding functions in Banach spaces with weak topology; Benedetti, Taddei and Väth [?] for sufficient conditions for invariant sets for nonlocal semilinear differential inclusions.

The approximation solvability method: As already mentioned, one of the most effective tools for the investigation of the solvability of equations in Banach spaces is the topological method suggested by Leray and Schauder [?]. However, from the practical point of view, it is often more important to study approximable solutions rather than usual solutions since the former ones can be localized by using the approximation methods. For simple description of approximation solvability method, we consider a separable Hilbert space $H$ with a basis $\left\{e_{i}\right\}_{i=1}^{\infty}$, then, denoting with $H_{n}$ the subspace with base $\left\{e_{1}, \cdots, e_{n}\right\}$, we approximate the original problem by a family of auxiliary problems by means of the natural projections $P_{n}: H \rightarrow H_{n}$ $(n \in \mathbb{N})$. Precisely, for a given $n \in \mathbb{N}$, we prove the existence of a solution in the space $W^{1,1}\left(I, H_{n}\right)$ for the problem

$$
\left\{\begin{array}{l}
x^{\prime}(t)=P_{n} f(t, x(t)) \text { for a.e. } t \in I, \\
x(0)=P_{n} M x
\end{array}\right.
$$

Then, by a limit argument, we obtain the existence of a solution for the original problem.

About the idea of combining these methods: A joint application of the LeraySchauder continuation principle with the bounding functions method was recently proposed also in reflexive Banach spaces (see e.g. [?] for the study of the Floquet problem and [?] for the investigation of nonlocal conditions). A regularity assumption is needed, in this context, which is expressed in terms of the Hausdorff measure of noncompactness $\chi$ (usually denoted $\chi$-regularity). A further restriction is required, essentially involving the rate of noncompactness of the model (see e.g. [?, condition (5.2)]).

We point out the fact that we prove the existence of a solution of problem (??) without any assumption of monotonicity or compactness nether on the nonlinearity $f$, nor on the nonlocal operator $M$. This can be done by the compact embedding of 
6 Benedetti, Loi, Malaguti and Obukhovskii

$H$ into $E$ and by the assumption of the continuity of $f(t, \cdot): H \rightarrow H$ for a.e. $t \in I$ w.r.t. the topology of $E$, see hypothesis $(f 2)$ below.

\section{Preliminaries and Notation}

Let $\mathcal{X}, \mathcal{Z}$ be Banach spaces. A map $\Sigma: \mathcal{X} \rightarrow \mathcal{Z}$ is said to be completely continuous if it is continuous and maps every bounded subset $U \subset \mathcal{X}$ into a relatively compact subset of $\mathcal{Z}$. Let us recall that if $U$ is an open bounded subset of $\mathcal{X}$ and $F: \bar{U} \rightarrow \mathcal{X}$ is a completely continuous map such that $x \neq F(x)$ for all $x \in \partial U$, then for the corresponding vector field $i-F$ (where $i$ denotes the inclusion map) the LeraySchauder topological degree $\operatorname{deg}(i-F, \bar{U})$ is well-defined (see, e.g. [?,?]).

In the sequel, by $\left(H,\|\cdot\|_{H}\right)$ we denote a separable Hilbert space which is compactly embedded into a Banach space $\left(E,\|\cdot\|_{E}\right)$ with the following relation between norms:

$$
\|w\|_{E} \leq q\|w\|_{H} \text { for all } w \in H, q>0 .
$$

Let $\left\{e_{n}\right\}_{n=1}^{\infty}$ be the orthonormal basis of $H$ and for every $n \in \mathbb{N}$, let $H_{n}$ be the $n$-dimensional subspaces of $H$ with the bases $\left\{e_{k}\right\}_{k=1}^{n}$ and $P_{n}$ be the natural projections of $H$ onto $H_{n}$. By $\langle\cdot, \cdot\rangle_{H}$ we denote the inner product in $H$. The open ball of radius $r$ centered at $x_{0} \in H\left[x_{0} \in E\right]$ in the space $H[E]$ is denoted by $B_{H}\left(x_{0}, r\right)$ [respectively $\left.B_{E}\left(x_{0}, r\right)\right]$. Put

$$
B_{H}(0, r, R)=\left\{w \in H: r<\|w\|_{H}<R\right\} .
$$

Throughout the paper, $I=[0, T]$ and let $C(I, H)\left[L^{1}(I, H)\right]$ be the space of all continuous [respectively integrable] functions $u: I \rightarrow H$ with usual norms

$$
\|u\|_{C}=\max _{t \in I}\|u(t)\|_{H} \quad \text { and } \quad\|u\|_{1}=\int_{0}^{T}\|u(t)\|_{H} d t .
$$

Consider the space of all absolutely continuous functions $u: I \rightarrow H$ whose generalized derivatives $u^{\prime}$ belong to $L^{1}(I, H)$. It is well known (see, e.g. [?]) that this space can be identified with the Sobolev space $W^{1,1}(I, H)$ endowed with the norm

$$
\|u\|_{W}=\|u\|_{1}+\left\|u^{\prime}\right\|_{1}
$$

and the embedding $W^{1,1}(I, H) \hookrightarrow C(I, H)$ is continuous.

Definition 3.1. Let $S \subseteq \mathbb{R}$ be a bounded and measurable subset. A subset $A \subset$ $L^{1}(S, H)$ is said uniformly integrable if for every $\epsilon>0$ there is $\delta>0$ such that $\Omega \subset S$ and $\mu(\Omega)<\delta$ implies

$$
\left\|\int_{\Omega} f d \mu\right\|<\epsilon \quad \text { for all } f \in A
$$

where $\mu$ is the Lebesgue measure on $\mathbb{R}$.

The following weak compactness criterion immediately follows from [?], p.101. 
Theorem 3.1. Let $A \subset L^{1}(S, H)$ be a bounded, uniformly integrable subset. Then $A$ is weakly relatively compact in $L^{1}(S, H)$.

For each $n \in \mathbb{N}$ define the map $\mathbb{P}_{n}: L^{1}(I, H) \rightarrow L^{1}\left(I, H_{n}\right)$ by

$$
\left(\mathbb{P}_{n} f\right)(t)=P_{n} f(t), t \in I .
$$

\section{Main result}

To study problem (??)-(??) we assume that:

( $f 1)$ the function $f: I \times H \rightarrow H$ is (globally) measurable while the measure on $I \times H$ is the product of the Lebesgue measure on $\mathbb{R}$ and the Borel measure on $H$;

( $f 2$ ) for a.e. $t \in I$ the map $f(t, \cdot): H \rightarrow H$ is $E-E$ continuous in the following sense: for each $w \in H$, and $\varepsilon>0$ there exists $\delta>0$ such that from $w^{\prime} \in B_{E}(w, \delta)$ it follows that $f\left(t, w^{\prime}\right) \in B_{E}(f(t, w), \varepsilon)$;

(f3) for every bounded subset $\Omega \subset H$ there exists a function $v_{\Omega} \in L_{+}^{1}[0, T]$ such that for each $\omega \in \Omega$ we have

$$
\|f(t, \omega)\|_{H} \leq v_{\Omega}(t)
$$

for a.e. $t \in I$;

(M) $M: C(I, H) \rightarrow H$ is a linear bounded operator such that $\|M\| \leq 1$.

Remark 4.1. (a) Condition $(f 1)$ can be easily obtained if $f$ is a Carathéodory map, i.e., for every $w \in H$ the function $f(\cdot, w): I \rightarrow H$ is measurable and for a.e. $t \in I$ the map $f(t, \cdot): H \rightarrow H$ is continuous.

(b) The class of boundary value problems with the operator $M$ satisfying condition $(M)$ is sufficiently large. In particular, it includes the following well-known problems:

(i) $M x=0$ (the general Cauchy condition $x(0)=x_{0}$ can be replaced by condition $z(0)=0$ by a transformation $\left.z=x-x_{0}\right)$;

(ii) $M x= \pm x(T)$ (periodic and anti-periodic problems);

(iii) $M x=\frac{1}{T} \int_{0}^{T} x(t) d t$ (mean value problem);

(iv) $M x=\sum_{i=1}^{k_{0}} \alpha_{i} x\left(t_{i}\right)$ with $\alpha_{i} \in \mathbb{R}$ and $\sum_{i=1}^{k_{0}}\left|\alpha_{i}\right| \leq 1$, where $0<t_{1}<\cdots<t_{k_{0}} \leq T$ (multi-point problem).

From conditions $(f 1)$ and $(f 3)$ it follows that for every $x \in C(I, H)$ the superposition function $f(s, x(s))$ belongs to $L^{1}(I, H)$.

By a solution to problem (??)-(??) we mean a function $x \in W^{1,1}(I, H)$ that satisfies (??)-(??).

The main result of this paper is the following statement.

Theorem 4.1. Let conditions $(f 1)-(f 3)$ and $(M)$ hold. In addition, assume that 
8 Benedetti, Loi, Malaguti and Obukhovskii

(f4) there exist $R_{0}>r_{0}>0$ such that

$$
\langle w, f(t, w)\rangle_{H}<0,
$$

for every $w \in B_{H}\left(0, r_{0}, R_{0}\right)$ and a.e. $t \in I$.

Then problem (??)-(??) admits a solution with values in $\overline{B_{H}\left(0, R_{0}\right)}$.

Proof. SteP 1. It is clear that for each $n \in \mathbb{N}$ problem

$$
\left\{\begin{array}{l}
x^{\prime}(t)=0, \text { for a.e. } t \in I, \\
x(0)=0,
\end{array}\right.
$$

has only the trivial solution in the space $W^{1,1}\left(I, H_{n}\right)$.

We are going to show now that, for a given $n \in \mathbb{N}$, the problem

$$
\left\{\begin{array}{l}
x^{\prime}(t)=P_{n} f(t, x(t)) \text { for a.e. } t \in I, \\
x(0)=P_{n} M x,
\end{array}\right.
$$

has a solution in the space $W^{1,1}\left(I, H_{n}\right)$.

To this aim, we choose arbitrarily $r_{*} \in\left(r_{0}, R_{0}\right)$ and let $\bar{K}=\overline{B_{H}\left(0, r_{*}\right)}, Q=C(I, \bar{K})$ and $Q^{(n)}=Q \cap C\left(I, H_{n}\right)$.

For each $y \in Q^{(n)}$ and $\lambda \in[0,1]$, the Cauchy problem

$$
\left\{\begin{array}{l}
x^{\prime}(t)=\lambda P_{n} f(t, y(t)) \text { for a.e. } t \in I, \\
x(0)=\lambda P_{n} M y
\end{array}\right.
$$

has a unique solution $x_{n} \in W^{1,1}\left(I, H_{n}\right)$ :

$$
x_{n}(t)=\lambda P_{n} M y+\lambda \int_{0}^{t} P_{n} f(s, y(s)) d s, t \in I .
$$

Define the map $\mathcal{T}_{n}: Q^{(n)} \times[0,1] \rightarrow C\left(I, H_{n}\right)$, assuming that $\mathcal{T}_{n}(y, \lambda)$ is the solution of (??). It is clear that

$$
\mathcal{T}_{n}\left(Q^{(n)} \times\{0\}\right)=\bigcup_{y \in Q^{(n)}} \mathcal{T}_{n}(y, 0)=\{0\} \subset \operatorname{int} Q^{(n)},
$$

where int $Q^{(n)}$ denotes the interior of $Q^{(n)}$.

SteP 2. (a) At first, let us show that the map $\mathcal{T}_{n}$ has a closed graph in the space $Q^{(n)} \times[0,1] \times C\left(I, H_{n}\right)$. Assume that

$$
\left(y^{(m)}, \lambda^{(m)}, x^{(m)}\right) \rightarrow\left(y^{(0)}, \lambda^{(0)}, x^{(0)}\right) \in Q^{(n)} \times[0,1] \times C\left(I, H_{n}\right),
$$

where $x^{(m)}=\mathcal{T}_{n}\left(y^{(m)}, \lambda^{(m)}\right)$. Then

$$
x^{(m)}(t)=\lambda^{(m)} P_{n} M y^{(m)}+\lambda^{(m)} \int_{0}^{t} P_{n} f\left(s, y^{(m)}(s)\right) d s, t \in I .
$$


Since $H$ is embedded in $E$, from condition $(f 2)$ we have

$$
P_{n} f\left(s, y^{(m)}(s)\right) \stackrel{E}{\rightarrow} P_{n} f\left(s, y^{(0)}(s)\right) \text { for a.e. } s \in I .
$$

The convergence is also dominated by ( $f 3)$. Passing to the limit $m \rightarrow \infty$ in (??) we obtain

$$
x^{(0)}(t)=\lambda^{(0)} P_{n} M y^{(0)}+\lambda^{(0)} \int_{0}^{t} P_{n} f\left(s, y^{(0)}(s)\right) d s, t \in I,
$$

i.e., $x^{(0)}=\mathcal{T}_{n}\left(y^{(0)}, \lambda^{(0)}\right)$.

(b) Now we will show that the set $\mathcal{T}_{n}\left(Q^{(n)} \times[0,1]\right)$ is relatively compact in $C\left(I, H_{n}\right)$. In fact, from $(f 3)$ and the boundedness of the set $Q^{(n)}$ it follows that the set $\mathcal{T}_{n}\left(Q^{(n)} \times[0,1]\right)$ is bounded and equicontinuous in $C\left(I, H_{n}\right)$, and therefore, it is relatively compact in $C\left(I, H_{n}\right)$. So, $\mathcal{T}_{n}$ is a closed and compact map, and therefore, it is completely continuous.

(c) Assume that there exists $\left(y_{n}, \lambda\right) \in \partial Q^{(n)} \times(0,1)$ such that $y_{n}=\mathcal{T}_{n}\left(y_{n}, \lambda\right)$. Then, $y_{n}(0)=\lambda P_{n} M y_{n}$ and

$$
y_{n}^{\prime}(t)=\lambda P_{n} f\left(t, y_{n}(t)\right) \text { for a.e. } t \in I .
$$

Since $y_{n} \in \partial Q^{(n)}$ we can choose $t_{0} \in[0, T]$ such that $\left\|y_{n}\left(t_{0}\right)\right\|_{H}=r_{*}$. Assume that $t_{0}=0$. Then

$$
r_{*}=\left\|y_{n}(0)\right\|_{H}=\lambda\left\|P_{n} M y_{n}\right\|_{H} \leq \lambda\left\|y_{n}\right\|_{C}<r_{*},
$$

that is a contradiction.

So, $t_{0} \in(0, T]$. Therefore, we can choose a sufficiently small $\varepsilon>0$ such that

$$
r_{0}<\left\|y_{n}(t)\right\|_{H} \leq r_{*}<R_{0} \text { for all } t \in\left(t_{0}-\varepsilon, t_{0}\right) .
$$

From the last inequalities it follows that

$$
\left\langle y_{n}(t), f\left(t, y_{n}(t)\right)\right\rangle_{H}<0 \text {, for a.e. } t \in\left(t_{0}-\varepsilon, t_{0}\right) .
$$

Since $y_{n}(t) \in H_{n}$ for all $t \in I$ we obtain

$$
\left\langle y_{n}(t), \lambda P_{n} f\left(t, y_{n}(t)\right)\right\rangle_{H}=\lambda\left\langle y_{n}(t), f\left(t, y_{n}(t)\right)\right\rangle_{H}<0
$$

for a.e. $t \in\left(t_{0}-\varepsilon, t_{0}\right)$.

Consequently,

$$
0>\int_{t_{0}-\varepsilon}^{t_{0}}\left\langle y_{n}(t), \lambda P_{n} f\left(t, y_{n}(t)\right)\right\rangle_{H} d t=\int_{t_{0}-\varepsilon}^{t_{0}}\left\langle y_{n}(t), y_{n}^{\prime}(t)\right\rangle_{H} d t \geq 0,
$$

giving the contradiction.

Thus, if there is $y_{n} \in \partial Q^{(n)}$ such that $y_{n}=\mathcal{T}_{n}\left(y_{n}, 1\right)$, then $y_{n}$ is a solution to (??). If $y_{n} \neq \mathcal{T}_{n}\left(y_{n}, 1\right)$ for all $y_{n} \in \partial Q^{(n)}$, then $\mathcal{T}_{n}$ is a homotopy connecting the 
maps $\mathcal{T}_{n}(\cdot, 0)$ and $\mathcal{T}_{n}(\cdot, 1)$. By virtue of the homotopy invariance and normalization properties of the topological degree we have

$$
\operatorname{deg}\left(i-\mathcal{T}_{n}(\cdot, 1), Q^{(n)}\right)=\operatorname{deg}\left(i-\mathcal{T}_{n}(\cdot, 0), Q^{(n)}\right)=1 .
$$

So, for every $n \in \mathbb{N}$ there exists $y_{n} \in Q^{(n)}$ such that $y_{n}=\mathcal{T}_{n}\left(y_{n}, 1\right)$, and hence $y_{n}$ satisfies (??).

SteP 3. Denote $f_{n}(t)=f\left(t, y_{n}(t)\right)$. From the condition $\left\|y_{n}(t)\right\|_{H} \leq r_{*}$ and according to $(f 3)$ it follows that there exists $\nu_{*} \in L^{1}(I, H)$ such that $\left\|f_{n}(t)\right\|_{H} \leq$ $\nu_{*}(t)$ for a.e. $t \in I$ and $n$. Therefore, the sequence $\left\{f_{n}\right\}$ is bounded and uniformly integrable in $L^{1}(I, H)$. By virtue of Theorem ?? it is relatively weakly compact in $L^{1}(I, H)$. W.l.o.g. assume that $f_{n} \stackrel{L^{1}(\underline{I}, H)}{{ }^{\prime}} f_{0}$.

Since $\left\|P_{n} f_{n}(t)\right\|_{H} \leq\left\|f_{n}(t)\right\|_{H} \leq \nu_{*}(t)$ for all $t \in I$, we can repeat previous reasoning also for $\left\{\mathbb{P}_{n} f_{n}=y_{n}^{\prime}\right\}$ and obtain that the set $\left\{y_{n}^{\prime}\right\}$ is weakly relatively compact in $L^{1}(I, H)$. Again w.l.o.g. we assume that

$$
\mathbb{P}_{n} f_{n}=y_{n}^{\prime} \stackrel{L^{1}\left(I_{\longrightarrow} H\right)}{y_{0}^{\prime}} .
$$

The set $\left\{y_{n}(0): n \in \mathbb{N}\right\}$ is bounded in $H$. So, w.l.o.g. we can assume that

$$
y_{n}(0) \stackrel{H}{\rightarrow} \gamma_{0} \text {. }
$$

Define

$$
y_{0}(t):=\gamma_{0}+\int_{0}^{t} y_{0}^{\prime}(s) d s, \quad t \in I .
$$

It is easy to see that

$$
y_{n}(t)=y_{n}(0)+\int_{0}^{t} y_{n}^{\prime}(s) d s \stackrel{H}{\rightarrow} y_{0}(t)
$$

for all $t \in I$.

Moreover, $\left\|y_{n}(t)\right\|_{H} \leq r_{*}$ for all $t$ and $n$. Therefore (see, e.g. [?])

$$
y_{n} \stackrel{C(I, H)}{\rightarrow} y_{0},
$$

and hence, $M y_{n} \stackrel{H}{\rightarrow} M y_{0}$.

For every $w \in H$, since $P_{n} w \stackrel{H}{\rightarrow} w$, we have

$$
\begin{gathered}
\left\langle P_{n} M y_{n}-M y_{0}, w\right\rangle_{H}=\left\langle P_{n} M y_{0}-M y_{0}, w\right\rangle_{H}+\left\langle P_{n} M y_{n}-P_{n} M y_{0}, w\right\rangle_{H} \\
=\left\langle P_{n} M y_{0}-M y_{0}, w\right\rangle_{H}+\left\langle M y_{n}-M y_{0}, P_{n} w\right\rangle_{H} \\
=\left\langle P_{n} M y_{0}-M y_{0}, w\right\rangle_{H}+\left\langle M y_{n}-M y_{0}, P_{n} w-w\right\rangle_{H}+\left\langle M y_{n}-M y_{0}, w\right\rangle_{H} .
\end{gathered}
$$

Therefore, $\left\langle P_{n} M y_{n}-M y_{0}, w\right\rangle_{H} \rightarrow 0$ as $n \rightarrow \infty$.

Consequently, $y_{n}(0)=P_{n} M y_{n} \stackrel{H}{\rightarrow} M y_{0}$. From (??) we obtain $y_{0}(0)=M y_{0}$.

On the other hand, the weak convergence $y_{n}(t) \rightarrow y_{0}(t)$ in $H$ for all $t$ implies 


$$
y_{n}(t) \stackrel{E}{\longrightarrow} y_{0}(t) \text { for every } t \in I \text {. }
$$

By virtue of the Lebesgue dominated convergence theorem we have

$$
\mathbb{P}_{n} g \stackrel{L^{1}(I, H)}{\longrightarrow} g
$$

for every element $g \in L^{1}(I, H)$.

Now we prove that $\mathbb{P}_{n} f_{n}{\stackrel{L^{1}}{\underline{(I, H)}}}$. To this aim, let $\Phi: L^{1}(I, H) \rightarrow \mathbb{R}$ be a linear and bounded functional. Hence, there is $\varphi \in L^{\infty}(I, H)$ such that

$$
\Phi(g)=\int_{0}^{T}\langle g(t), \varphi(t)\rangle_{H} d t \text { for all } g \in L^{1}(I, H) .
$$

We have

$$
\begin{aligned}
\Phi & \left(\mathbb{P}_{n} f_{n}-f_{0}\right)=\int_{0}^{T}\left\langle P_{n} f_{n}(t)-f_{0}(t), \varphi(t)\right\rangle_{H} d t \\
= & \int_{0}^{T}\left\langle P_{n} f_{n}(t)-P_{n} f_{0}(t), \varphi(t)\right\rangle_{H} d t+\int_{0}^{T}\left\langle P_{n} f_{0}(t)-f_{0}(t), \varphi(t)\right\rangle_{H} d t \\
= & \int_{0}^{T}\left\langle f_{n}(t)-f_{0}(t), P_{n} \varphi(t)\right\rangle_{H} d t+\int_{0}^{T}\left\langle P_{n} f_{0}(t)-f_{0}(t), \varphi(t)\right\rangle_{H} d t \\
= & \int_{0}^{T}\left\langle f_{n}(t)-f_{0}(t), \varphi(t)\right\rangle_{H} d t+\int_{0}^{T}\left\langle f_{n}(t)-f_{0}(t), P_{n} \varphi(t)-\varphi(t)\right\rangle_{H} d t \\
& +\int_{0}^{T}\left\langle P_{n} f_{0}(t)-f_{0}(t), \varphi(t)\right\rangle_{H} d t \\
= & \Phi\left(f_{n}-f_{0}\right)+\int_{0}^{T}\left\langle f_{n}(t)-f_{0}(t), P_{n} \varphi(t)-\varphi(t)\right\rangle_{H} d t+\Phi\left(\mathbb{P}_{n} f_{0}-f_{0}\right) .
\end{aligned}
$$

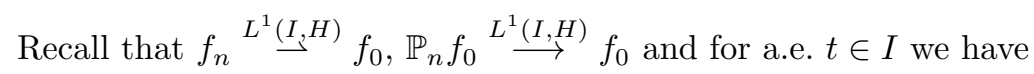

$$
\begin{aligned}
\left|\left\langle f_{n}(t)-f_{0}(t), P_{n} \varphi(t)-\varphi(t)\right\rangle_{H}\right| & \leq\left\|f_{n}(t)-f_{0}(t)\right\|_{H} \cdot\left\|P_{n} \varphi(t)-\varphi(t)\right\|_{H} \\
& \leq 2\left(v_{*}(t)+\left\|f_{0}(t)\right\|_{H}\right)\|\varphi(t)\|_{H},
\end{aligned}
$$

and

$$
\lim _{n \rightarrow \infty}\left\|P_{n} \varphi(t)-\varphi(t)\right\|_{H}=0 .
$$

Therefore, for a.e. $t \in I$ we have

$$
\lim _{n \rightarrow \infty}\left\langle f_{n}(t)-f_{0}(t), P_{n} \varphi(t)-\varphi(t)\right\rangle_{H}=0 .
$$

Moreover, the function $g_{*}(t):=\left(v_{*}(t)+\left\|f_{0}(t)\right\|_{H}\right)\|\varphi(t)\|_{H}, t \in I$, is integrable since

$$
\int_{0}^{T}\left\|g_{*}(t)\right\|_{H} d t \leq\|\varphi\|_{\infty} \int_{0}^{T}\left(v_{*}(t)+\left\|f_{0}(t)\right\|_{H}\right) d t
$$


where $\|\varphi\|_{\infty}$ is the norm of $\varphi$ in $L^{\infty}(I, H)$.

From the Lebesgue dominated convergence theorem it follows that

$$
\int_{0}^{T}\left\langle f_{n}(t)-f_{0}(t), P_{n} \varphi(t)-\varphi(t)\right\rangle_{H} d t \rightarrow 0 \text { as } n \rightarrow \infty .
$$

Therefore, $\Phi\left(\mathbb{P}_{n} f_{n}-f_{0}\right) \rightarrow 0$ as $n \rightarrow \infty$, i.e., $\mathbb{P}_{n} f_{n} \stackrel{L^{1}(I, H)}{\longrightarrow} f_{0}$. So, from (??) we conclude that $y_{0}^{\prime}=f_{0}$, and hence, $f_{n} \stackrel{L^{1}\left(I_{2} H\right)}{\prime} y_{0}^{\prime}$. By the Mazur Lemma (see, e.g. [?], p. 16) there is a sequence of convex combinations $\left\{\bar{f}^{(n)}\right\}$,

$$
\bar{f}^{(n)}=\sum_{k=n}^{\infty} \sigma_{n k} f_{k}, \sigma_{n k} \geq 0 \text { and } \sum_{k=n}^{\infty} \sigma_{n k}=1,
$$

which converges to $y_{0}^{\prime}$ in $L^{1}(I, H)$. Applying e.g. [?, Theorem 38] we assume w.l.o.g that $\left\{\bar{f}^{(n)}\right\}$ converges to $y_{0}^{\prime}$ for a.e. $t \in I$.

From the embedding $H \hookrightarrow E$ we have that

$$
\bar{f}^{(n)}(t) \stackrel{E}{\rightarrow} y_{0}^{\prime}(t) \text { for a.e. } t \in I .
$$

Applying (??) and condition $(f 2)$ we obtain that for a.e. $t \in I$ and every $\varepsilon>0$ there is an integer $i_{0}=i_{0}(\varepsilon, t)$ such that

$$
f\left(t, y_{i}(t)\right) \in B_{E}\left(f\left(t, y_{0}(t)\right), \varepsilon\right) \text { for all } i \geq i_{0} .
$$

So, $f_{i}(t) \in B_{E}\left(f\left(t, y_{0}(t)\right), \varepsilon\right)$ for all $i \geq i_{0}$, and by the convexity of the set $B_{E}\left(f\left(t, y_{0}(t)\right), \varepsilon\right)$ we have

$$
\bar{f}^{(n)}(t) \in B_{E}\left(f\left(t, y_{0}(t)\right), \varepsilon\right) \text { for all } n \geq i_{0} .
$$

Therefore, $y_{0}^{\prime}(t)=f\left(t, y_{0}(t)\right)$ for a.e. $t \in I$. Combining with $y_{0}(0)=M y_{0}$ we obtain that $y_{0}$ is a solution to problem (??)-(??).

Remark 4.2. Theorem ?? gives us not only the existence of a solution $y_{0}$ to problem (??)-(??). It also provides us an important information about this solution, that is the (weak) convergence of the sequence $\left\{y_{n}\right\}$ to $y_{0}$. Since each $y_{n}$ takes its values in a finite-dimensional subspace, we can approximate the solution $y_{0}$ by finite-dimensional functions via weak approximation scheme.

\section{Applications to semilinear differential equations in Hilbert spaces}

Consider the following semilinear differential equation

$$
\left\{\begin{array}{l}
x^{\prime}(t)+A x(t)=f(t, x(t)) \text { for a.e. } t \in I \\
x(0)=M x
\end{array}\right.
$$


where $A: H \rightarrow H$ is a bounded linear operator which is $E-E$ continuous; $f$ and $M$ satisfy $(f 1)-(f 3)$ and $(M)$, respectively.

Theorem 5.1. If there exist $R_{0}>r_{0}>0$ such that for all $w \in B_{H}\left(0, r_{0}, R_{0}\right)$ and a.e. $t \in I$ relation

$$
\langle w,-A w+f(t, w)\rangle_{H}<0
$$

is satisfied, then problem (??) has a solution.

Proof. Problem (??) can be substituted with the following problem

$$
\left\{\begin{array}{l}
x^{\prime}(t)=\widetilde{f}(t, x(t)), \text { for a.e. } t \in I, \\
x(0)=M x,
\end{array}\right.
$$

where $\widetilde{f}: I \times H \rightarrow H$,

$$
\widetilde{f}(t, w)=-A w+f(t, w) .
$$

It is easy to verify that problem (??) satisfies all conditions in Theorem ??, therefore it, and hence problem (??), has a solution.

In order to illustrate the result, we consider two integro-differential equations. The first one is considered on an open bounded domain $\Omega \subset \mathbb{R}^{k}(k \geq 2)$ with Lipschitz boundary (Example ??), whereas the second one is defined on a given interval (Example ??).

Example 5.1. Consider the periodic problem

$$
\left\{\begin{array}{l}
u_{t}+a \int_{\Omega} u(t, \xi) d \xi=-b u(t, \xi)+f(t, u(t, \xi)), \\
u(0, \xi)=u(1, \xi)
\end{array}\right.
$$

for a.e. $t \in[0,1]$ and all $\xi \in \Omega$, where $a, b>0$ and $f:[0,1] \times \mathbb{R} \rightarrow \mathbb{R}$ is a continuous map.

Assume that

$(f 1)^{\prime}$ the partial derivative $\frac{\partial f}{\partial z}:[0,1] \times \mathbb{R} \rightarrow \mathbb{R}$ is (globally) continuous;

$(f 2)^{\prime}$ there is a positive number $N<b$ such that

$$
\left|\frac{\partial f(t, z)}{\partial z}\right| \leq N \text { for all }(t, z) \in[0,1] \times \mathbb{R} .
$$

By a solution to (??) we mean a continuous function $u:[0,1] \times \Omega \rightarrow \mathbb{R}$ whose partial derivative $\frac{\partial u(t, \xi)}{\partial t}$ exists and satisfies (??). Moreover, we can consider relation (??) as the law of evolution of a dynamical system with the state function $u(t, \xi)$. Our goal can be formulated as finding of the dynamics of the system as the continuous function $u(t, \xi)$ such that at every value $t$ the function $u(t, \cdot)$ belongs to the Sobolev space $W^{1,2}(\Omega)$. 
Theorem 5.2. Let conditions $(f 1)^{\prime}-(f 2)^{\prime}$ be satisfied. Then problem (??) has a solution. Moreover, if $f(t, 0) \neq 0$ for all $t \in[0,1]$, then the solution is non-zero.

Proof. Let $H=W^{1,2}(\Omega)$ and $E=L^{2}(\Omega)$. It is clear that $H$ is a separable Hilbert space which is compactly embedded in $E$ and for every $w \in H$ :

$$
\|w\|_{H}=\sqrt{\|w\|_{2}^{2}+\|D w\|_{2}^{2}}
$$

where $\|w\|_{2}^{2}=\int_{\Omega} w^{2}(\xi) d \xi$ and $D$ denotes the derivative (i.e. gradient) of a function with several variables.

For each $t \in[0,1]$, set $x(t)=u(t, \cdot)$. Then we can substitute $(? ?)$ with the following problem

$$
\left\{\begin{array}{l}
x^{\prime}(t)+A x(t)=\bar{f}(t, x(t)), \text { for a.e. } t \in[0,1] \\
x(0)=x(1)
\end{array}\right.
$$

where $A: H \rightarrow H, A w=a \int_{\Omega} w(\xi) d \xi$, and

$$
\bar{f}:[0,1] \times H \rightarrow H, \quad \bar{f}(t, w)(\xi)=-b w(\xi)+f(t, w(\xi)) .
$$

Notice that the map $\bar{f}$ is well-defined since

$$
D \bar{f}(t, w)(\xi)=-b D w(\xi)+f_{2}^{\prime}(t, w(\xi)) D w(\xi),
$$

where $f_{2}^{\prime}=\frac{\partial f}{\partial z}$. From $(f 1)^{\prime}-(f 2)^{\prime}$ it follows that

$$
|f(t, z)|=\left|f(t, 0)+f_{2}^{\prime}(t, \eta) z\right| \leq|f(t, 0)|+N|z|
$$

for all $(t, z) \in[0,1] \times \mathbb{R}$, where $\eta$ is a number between 0 and $z$.

It is easy to verify that $A$ is a linear bounded operator which is $E-E$ continuous. Let us show that the map $\bar{f}(t, \cdot)$ satisfies condition $(f 2)$.

Let $\left\{w_{n}\right\}$ in $H$ be such that $w_{n} \stackrel{E}{\rightarrow} w_{0}$. By $(f 1)^{\prime}$ for every $(t, \xi) \in(0,1) \times \Omega$ we have

$$
\left|f\left(t, w_{n}(\xi)\right)-f\left(t, w_{0}(\xi)\right)\right|=\left|f_{2}^{\prime}(t, \eta) \cdot\left(w_{n}(\xi)-w_{0}(\xi)\right)\right| \leq N\left|w_{n}(\xi)-w_{0}(\xi)\right|,
$$

where $\eta$ is a number between $w_{n}(\xi)$ and $w_{0}(\xi)$.

Therefore,

$$
\begin{aligned}
\left\|\bar{f}\left(t, w_{n}\right)-\bar{f}\left(t, w_{0}\right)\right\|_{E}^{2} & =\int_{\Omega}\left|-b w_{n}(\xi)+f\left(t, w_{n}(\xi)\right)+b w_{0}(\xi)-f\left(t, w_{0}(\xi)\right)\right|^{2} d \xi \\
& \leq 2\left(b^{2}+N^{2}\right) \int_{\Omega}\left|w_{n}(\xi)-w_{0}(\xi)\right|^{2} d \xi \\
& \leq 4 b^{2}\left\|w_{n}-w_{0}\right\|_{E}^{2} .
\end{aligned}
$$

So, $\bar{f}\left(t, w_{n}\right) \stackrel{E}{\rightarrow} \bar{f}\left(t, w_{0}\right)$, and hence, condition $(f 2)$ is satisfied. 
Let $U \subset H$ be a bounded set. For every $w \in U$ we have

$$
\begin{aligned}
\|\bar{f}(t, w)\|_{H}^{2}=\int_{\Omega}|-b w(\xi)+f(t, w(\xi))|^{2} d \xi & \\
& +\int_{\Omega}\left|-b D w(\xi)+f_{2}^{\prime}(t, w(\xi)) D w(\xi)\right|^{2} d \xi \\
\leq 2 b^{2} \int_{\Omega}|w(\xi)|^{2} d \xi & +2 \int_{\Omega}(|f(t, 0)|+N|w(\xi)|)^{2} d \xi \\
& +2\left(b^{2}+N^{2}\right) \int_{\Omega}|D w(\xi)|^{2} d \xi
\end{aligned}
$$

where for each $\xi \in \Omega, D w(\xi)$ is a vector in $\mathbb{R}^{k}$ and

$$
|D w(\xi)|^{2}=\langle D w(\xi), D w(\xi)\rangle .
$$

Consequently, condition $(f 3)$ is satisfied.

To verify condition $(f 1)$ we will apply the Pettis Measurability Theorem (see, e.g. [?]). Notice that $H$ can be identified with its dual space $H^{*}$. So, in order to apply Pettis Theorem, we have to prove that for every $\varphi \in H$ the $\operatorname{map} \bar{f}_{\varphi}:[0,1] \times H \rightarrow \mathbb{R}$, defined as

$$
\bar{f}_{\varphi}(t, w)=\langle\varphi, \bar{f}(t, w)\rangle_{H}
$$

is measurable. To this aim, we will prove that $\bar{f}_{\varphi}$ is a Carathéodory map.

Fix $w \in H$ and consider the map $\bar{f}_{\varphi}(\cdot, w):[0,1] \rightarrow \mathbb{R}$. Assume that there is a sequence $\left\{t_{n}\right\} \subset[0,1]$ such that $t_{n} \rightarrow t_{0} \in[0,1]$. Let $r_{n}=\bar{f}_{\varphi}\left(t_{n}, w\right)$ and $r_{0}=$ $\bar{f}_{\varphi}\left(t_{0}, w\right)$. From the continuity property of $f$ and $f_{2}^{\prime}$ we have $r_{n} \rightarrow r_{0}$. Therefore, $\bar{f}_{\varphi}(\cdot, w)$ is continuous, and hence, it is measurable.

We will prove now that for every $t \in[0,1]$ the map $\bar{f}_{\varphi}(t, \cdot): H \rightarrow \mathbb{R}$ is continuous. Let $\left\{w_{n}\right\} \subset H$ be such that $w_{n} \rightarrow w_{0} \in H$. Set $\gamma_{n}=\left\langle\bar{f}\left(t, w_{n}\right), \varphi\right\rangle_{H}$ and

$$
\lambda_{0}=\liminf _{n \rightarrow \infty} \gamma_{n} \text { and } \Lambda_{0}=\limsup _{n \rightarrow \infty} \gamma_{n} .
$$

Then there are subsequences $\left\{w_{n_{k}}\right\}$ and $\left\{w_{m_{k}}\right\}$ of $\left\{w_{n}\right\}$ such that

$$
\lambda_{0}=\lim _{k \rightarrow \infty}\left\langle\bar{f}\left(t, w_{n_{k}}\right), \varphi\right\rangle_{H} \quad \text { and } \quad \Lambda_{0}=\lim _{k \rightarrow \infty}\left\langle\bar{f}\left(t, w_{m_{k}}\right), \varphi\right\rangle_{H} .
$$

The sets $\left\{\bar{f}\left(t, w_{n_{k}}\right)\right\}$ and $\left\{\bar{f}\left(t, w_{m_{k}}\right)\right\}$ are bounded in $H$, and so they are weakly relatively compact. W.l.o.g. assume that

$$
\bar{f}\left(t, w_{n_{k}}\right) \stackrel{H}{\rightarrow} \bar{f}_{0} \quad \text { and } \quad \bar{f}\left(t, w_{m_{k}}\right) \stackrel{H}{\rightarrow} \bar{f}_{1} .
$$

Therefore,

$$
\lambda_{0}=\left\langle\bar{f}_{0}, \varphi\right\rangle_{H} \quad \text { and } \quad \Lambda_{0}=\left\langle\bar{f}_{1}, \varphi\right\rangle_{H}
$$

On the other hand, since $\bar{f}(t, \cdot)$ is $E-E$ continuous and

$$
w_{n_{k}} \stackrel{E}{\longrightarrow} w_{0} \stackrel{E}{\longleftarrow} w_{m_{k}} \text { as } k \rightarrow \infty
$$


we have

$$
\bar{f}\left(t, w_{n_{k}}\right) \stackrel{E}{\longrightarrow} \bar{f}\left(t, w_{0}\right) \stackrel{E}{\longleftarrow} \bar{f}\left(t, w_{m_{k}}\right) \text { as } k \rightarrow \infty .
$$

Consequently, $\bar{f}_{0}=\bar{f}_{1}$, and hence $\lambda_{0}=\Lambda_{0}$, i.e. $\bar{f}(t, \cdot)$ is continuous. Thus, the map $\bar{f}_{\varphi}$ is Carathéodory, and so, condition $(f 1)$ is satisfied.

Now for $w \in H$ and for a.e. $t \in[0,1]$ we have

$$
\langle w,-A w\rangle_{H}=-a\left(\int_{\Omega} w(\xi) d \xi\right)^{2} \leq 0
$$

By virtue of $(f 1)^{\prime}-(f 2)^{\prime}$ and (??) the following estimation is true

$$
\begin{aligned}
\langle w, \bar{f}(t, w)\rangle_{H}= & \int_{\Omega} w(\xi)(-b w(\xi)+f(t, w(\xi))) d \xi \\
& +\int_{\Omega} D w(\xi)\left(-b D w(\xi)+f_{2}^{\prime}(t, w(\xi)) D w(\xi)\right) d \xi \\
= & -b\left(\int_{\Omega}(w(\xi))^{2} d \xi+\int_{\Omega}(D w(\xi))^{2} d \xi\right) \\
& +\int_{\Omega} w(\xi) f(t, w(\xi)) d \xi+\int_{\Omega} D w(\xi) f_{2}^{\prime}(t, w(\xi)) D w(\xi) d \xi \\
\leq & -b\|w\|_{H}^{2}+\int_{\Omega}|w(\xi)|(|f(t, 0)|+N|w(\xi)|) d \xi \\
& +N \int_{\Omega}|D w(\xi)|^{2} d \xi \\
\leq & (-b+N)\|w\|_{H}^{2}+\beta|\Omega|\|w\|_{H}<0,
\end{aligned}
$$

provided $\|w\|_{H}>\frac{\beta|\Omega|}{b-N}$, where $\beta=\max _{[0,1]}|f(t, 0)|$ and $|\Omega|$ denotes the Lebesgue measure of $\Omega$.

Applying Theorem ?? we obtain the existence of a solution of (??), and therefore, problem (??) has a solution.

Example 5.2. Consider the mean value problem for an integro-differential equation of the form:

$$
\left\{\begin{array}{l}
u_{t}(t, s)+a u(t, s)=\int_{0}^{1} k(s, \xi) u(t, \xi) d \xi+f(t, u(t, s)), \\
u(0, s)=\int_{0}^{1} u(t, s) d t
\end{array}\right.
$$

for a.e. $t \in[0,1]$ and all $s \in[0,1]$, where $k(\cdot, \cdot) \in C^{1}([0,1] \times[0,1] ; \mathbb{R}) ; a>0$ and $f:[0,1] \times \mathbb{R} \rightarrow \mathbb{R}$ is a continuous map satisfying conditions $(f 1)^{\prime}-(f 2)^{\prime}$ (see Example ??).

Let $H=W^{1,2}[0,1]$ and $E=C[0,1]$. For each $t \in[0,1]$ set $x(t)=u(t, \cdot)$. Then problem (??) can be replaced with the following problem

$$
\left\{\begin{array}{l}
x^{\prime}(t)+A x(t)=\hat{f}(t, x(t)), \text { for a.e. } t \in[0,1], \\
x(0)=M x
\end{array}\right.
$$


where $M: C(I, H) \rightarrow H$,

$$
M x=\int_{0}^{1} x(t) d t
$$

$A: H \rightarrow H, A w=a w$,

and

$$
\hat{f}:[0,1] \times H \rightarrow H, \quad \hat{f}(t, w)(s)=\int_{0}^{1} k(s, \xi) w(\xi) d \xi+f(t, w(s)) .
$$

Notice that the map $\hat{f}$ is well-defined since

$$
\frac{d \hat{f}(t, w)(s)}{d s}=\int_{0}^{1} k_{1}^{\prime}(s, \xi) w(\xi) d \xi+f_{2}^{\prime}(t, w(s)) w^{\prime}(s),
$$

where $k_{1}^{\prime}=\frac{\partial k}{\partial s}$.

It is clear that $A$ is a linear bounded operator which is $E-E$ continuous and the operator $M$ satisfies condition $(M)$. The map $\hat{f}$ can be written as

$$
\hat{f}(t, w)=g(w)+\bar{f}(t, w),
$$

where

$$
\bar{f}:[0,1] \times H \rightarrow H, \quad \bar{f}(t, w)(\xi)=f(t, w(\xi))
$$

and $g: H \rightarrow H$ is defined by

$$
g(w)(s)=\int_{0}^{1} k(s, \xi) w(\xi) d \xi
$$

Notice that the map $\bar{f}$ differs from the one in Example ?? only by the linear term $b w, b \in \mathbb{R}$, here missing. Hence, as in the cited example it is possible to prove that satisfies conditions $(f 1)-(f 3)$. Let us show that the map $g$ satisfies conditions $(f 1)-(f 3)$.

At first, let $\left\{w_{n}\right\} \subset H$ be such that $w_{n} \stackrel{E}{\rightarrow} w_{0}$. We have

$$
\begin{aligned}
& \left\|g\left(w_{n}\right)-g\left(w_{0}\right)\right\|_{E}= \\
& =\max _{s \in[0,1]}\left|\int_{0}^{1} k(s, \xi) w_{n}(\xi) d \xi-\int_{0}^{1} k(s, \xi) w_{0}(\xi) d \xi\right| \\
& \leq \max _{s \in[0,1]} \int_{0}^{1}\left|k(s, \xi) \| w_{n}(\xi)-w_{0}(\xi)\right| d \xi \\
& \leq k\left\|w_{n}-w_{0}\right\|_{E},
\end{aligned}
$$

where $k=\max \{|k(s, \xi)|: s, \xi \in[0,1]\}$.

So, $g\left(w_{n}\right) \stackrel{E}{\rightarrow} g\left(w_{0}\right)$, and hence, condition $(f 2)$ is satisfied. 
Let $\mathcal{D} \subset H$ be a bounded set, for any $w \in \mathcal{D}$ we have

$$
\begin{aligned}
\|g(w)\|_{H}^{2} & =\int_{0}^{1}\left|\int_{0}^{1} k(s, \xi) w(\xi) d \xi\right|^{2} d s+\int_{0}^{1}\left|\int_{0}^{1} k_{1}^{\prime}(s, \xi) w(\xi) d \xi\right|^{2} d s \\
& \leq \int_{0}^{1} \int_{0}^{1} k^{2}(s, \xi) w^{2}(\xi) d \xi d s+\int_{0}^{1} \int_{0}^{1}\left(k_{1}^{\prime}(s, \xi)\right)^{2} w^{2}(\xi) d \xi d s \\
& \leq\left(k^{2}+k^{\prime 2}\right)\|w\|_{H}^{2} .
\end{aligned}
$$

where $k^{\prime}=\max \left\{\left|k_{1}^{\prime}(s, \xi)\right|: s, \xi \in[0,1]\right\}$.

Therefore, condition $(f 3)$ is satisfied.

To verify condition $(f 1)$, we prove that the map $g$ is $H-H$ continuous. For this, let $\left\{w_{n}\right\} \subset H$ be such that $w_{n} \stackrel{H}{\rightarrow} w_{0}$. We have

$$
\begin{aligned}
\left\|g\left(w_{n}\right)-g\left(w_{0}\right)\right\|_{H}^{2}= & \int_{0}^{1}\left|\int_{0}^{1} k(s, \xi)\left(w_{n}(\xi)-w_{0}(\xi)\right) d \xi\right|^{2} d s \\
& +\int_{0}^{1}\left|\int_{0}^{1} k_{1}^{\prime}(s, \xi)\left(w_{n}(\xi)-w_{0}(\xi)\right) d \xi\right|^{2} d s \\
\leq & k^{2}\left\|w_{n}-w_{0}\right\|_{2}^{2}+k^{\prime 2}\left\|w_{n}-w_{0}\right\|_{2}^{2} .
\end{aligned}
$$

Consequently, $g$ is $H-H$ continuous. So, condition $(f 1)$ is satisfied.

Now for $w \in H$ we have

$$
\begin{aligned}
&\langle w,-A w+\hat{f}(t, w)\rangle_{H}= \\
&=-a\|w\|_{H}^{2}+\int_{0}^{1} w(s)\left(\int_{0}^{1} k(s, \xi) w(\xi) d \xi\right) d s+\int_{0}^{1} w(s) f(t, w(s)) d s \\
&+\int_{0}^{1} w^{\prime}(s)\left(\int_{0}^{1} k_{1}^{\prime}(s, \xi) w(\xi) d \xi\right) d s+\int_{0}^{1} w^{\prime}(s) f_{2}^{\prime}(t, w(s)) w^{\prime}(s) d s \\
& \leq-a\|w\|_{H}^{2}+k\left(\int_{0}^{1}|w(\xi)| d \xi\right)^{2}+\int_{0}^{1}|w(s)|(|f(t, 0)|+N|w(s)|) d s \\
& \quad+k^{\prime} \int_{0}^{1}|w(\xi)| d \xi \int_{0}^{1}\left|w^{\prime}(s)\right| d s+N \int_{0}^{1}\left(w^{\prime}(s)\right)^{2} d s \\
& \leq-\left(a-k-\frac{k^{\prime}}{2}-N\right)\|w\|_{H}^{2}+\beta\|w\|_{H}<0
\end{aligned}
$$

provided $a>k+\frac{k^{\prime}}{2}+N$ and

$$
\|w\|_{H}>\frac{\beta}{a-k-\frac{k^{\prime}}{2}-N},
$$

where $N$ is the constant from $(f 2)^{\prime}$ and $\beta=\max _{t \in[0,1]}|f(t, 0)|$.

Hence, applying Theorem ?? we obtain

Theorem 5.3. If $a>k+\frac{k^{\prime}}{2}+N$, then problem (??) has a solution. 
Remark 5.1. Under similar assumptions we can consider problems (??) and (??) with various boundary conditions (periodic, anti-periodic, mean value or multi-point conditions).

\section{Existence of bounded solutions}

Consider now the Cauchy problem

$$
\left\{\begin{array}{l}
x^{\prime}(t)=f(t, x(t)) \text { for a.e. } t \in[0, \infty), \\
x(0)=0
\end{array}\right.
$$

where $f:[0, \infty) \times H \rightarrow H$ satisfies conditions $(f 1)-(f 3)$ with $[0, \infty)$ instead of $[0, T]$. In addition, assume that

$(f 4)$ there exist $R_{0}>r_{0}>0$ such that

$$
\langle w, f(t, w)\rangle_{H}<0
$$

for a.e. $t \in[0, \infty)$ and for all $w \in B_{H}\left(0, r_{0}, R_{0}\right)$.

Theorem 6.1. Under above assumptions, problem (??) has a solution $x:[0, \infty) \rightarrow$ $H$ such that $\|x(t)\|_{H} \leq R_{0}$ for all $t \in[0, \infty)$. Moreover, if $f(t, 0) \neq 0$ for a.e. $t \in[0, \infty)$, then the solution is non-zero.

Proof. According to Theorem ??, for every $n \in \mathbb{N}$ there exists a solution $x_{n}$ to the problem

$$
\left\{\begin{array}{l}
x^{\prime}(t)=f(t, x(t)) \text { for a.e. } t \in[0, n], \\
x(0)=0,
\end{array}\right.
$$

such that $\left\|x_{n}\right\|_{H} \leq R_{0}$ for all $t \in[0, n]$.

Define

$$
\widetilde{x}_{n}(t)=\left\{\begin{array}{l}
x_{n}(t) \text { for } t \in[0, n] \\
x_{n}(n) \text { for } t \geq n
\end{array}\right.
$$

According to $(f 3)$ there is $\gamma \in L_{+}^{1}[0, \infty)$ such that

$$
\left\|\widetilde{x}_{n}^{\prime}(t)\right\|_{H} \leq \gamma(t) \text { for a.e. } t \in[0, \infty) .
$$

It implies that the set $\left\{\widetilde{x}_{n}^{\prime}\right\}$ is bounded and uniformly integrable in $L^{1}([0, \infty) ; H)$. By applying Theorem ?? in the interval $[0,1]$ we obtain a subsequence $\left\{\widetilde{x}_{n}^{\prime(1)}\right\}$ satisfying

$$
\widetilde{x}_{n}^{\prime(1)} \stackrel{L^{1}}{([0,1] ; H)} y_{1}
$$

By an induction argument, for every $p>1$ it is possible to get a sequence $\left\{\widetilde{x}_{n}^{\prime(p)}\right\}$ and a function $y_{p} \in L^{1}([p-1, p] ; H)$ such that 
(i) $\left\{\widetilde{x}_{n}^{\prime(p)}\right\}$ is a subsequence of $\left\{\widetilde{x}_{n}^{\prime(p-1)}\right\}$;

(ii) $\widetilde{x}_{n}^{\prime(p)} \stackrel{L^{1}}{([p-1, p] ; H)} y_{p}$.

Let $x_{0}^{\prime}:[0,+\infty) \rightarrow H$ be given by $x_{0}^{\prime}(t)=: y_{p}(t)$ for $p-1 \leq t<p, p \in N_{0}$. Let us restrict our attention to $\left\{\widetilde{x}_{n}{ }^{(n)}\right\}$. Since

$$
\int_{p-1}^{p} \widetilde{x}_{n}^{\prime(n)}(\tau) d \tau \rightarrow \int_{p-1}^{p} y_{p}(\tau) d \tau, \quad p \in N_{0}
$$

for $t \geq 0$

$$
\widetilde{x}_{n}^{(n)}(t)=\int_{0}^{t} \widetilde{x}_{n}^{\prime(n)}(\tau) d \tau-\int_{0}^{t} x_{0}^{\prime}(\tau) d \tau:=x_{0}(t)
$$

From the compact embedding $H \hookrightarrow E$ we have

$$
\widetilde{x}_{n}^{(n)}(t) \stackrel{E}{\rightarrow} x_{0}(t), \text { for all } t>0 .
$$

Fix $t$ and take $n>t$. Notice that

$$
x_{n}(t)=\int_{0}^{t} f\left(\tau, x_{n}(\tau)\right) d \tau
$$

From $(f 2),(? ?)$ and since $\widetilde{x}_{n}{ }^{(n)}(\tau)=x_{n}{ }^{(n)}(\tau), \tau \in[0, t]$, it follows that

$$
f\left(\tau, x_{n}{ }^{(n)}(\tau)\right) \stackrel{E}{\rightarrow} f\left(\tau, x_{0}(\tau)\right) \text { for all } \tau \in[0, t] .
$$

By virtue of (??) we have

$$
\left\|f\left(\tau, x_{n}(\tau)\right)\right\|_{E} \leq q\left\|f\left(\tau, x_{n}(\tau)\right)\right\|_{H} \leq q \gamma(\tau)
$$

for a.e. $\tau \in[0, t]$.

Therefore, passing to the limit we obtain

$$
x_{0}(t) \stackrel{E}{\leftarrow} x_{n}{ }^{(n)}(t)=\int_{0}^{t} f\left(\tau, x_{n}{ }^{(n)}(\tau)\right) d \tau \stackrel{E}{\rightarrow} \int_{0}^{t} f\left(\tau, x_{0}(\tau)\right) d \tau .
$$

Thus, $x_{0}$ is a solution to problem (??) with $\left\|x_{0}(t)\right\|_{H} \leq R_{0}$ for all $t \in[0, \infty)$.

\section{Systems of differential equations}

Let $H_{i}(i=1,2)$ be separable Hilbert spaces which are compactly embedded in Banach spaces $E_{i}$, respectively. Consider the product spaces $H=H_{1} \times H_{2}$ and $E=E_{1} \times E_{2}$ with the norms:

$$
\|w\|_{H}=\sqrt{\left\|w_{1}\right\|_{H_{1}}^{2}+\left\|w_{2}\right\|_{H_{2}}^{2}}, \forall w=\left(w_{1}, w_{2}\right) \in H
$$

and

$$
\|w\|_{E}=\sqrt{\left\|w_{1}\right\|_{E_{1}}^{2}+\left\|w_{2}\right\|_{E_{2}}^{2}}, \forall w=\left(w_{1}, w_{2}\right) \in E
$$


It is clear that the embedding $H \hookrightarrow E$ is compact and $H$ is a separable Hilbert space with the inner product

$$
\langle w, \widetilde{w}\rangle_{H}=\left\langle w_{1}, \widetilde{w}_{1}\right\rangle_{H_{1}}+\left\langle w_{2}, \widetilde{w}_{2}\right\rangle_{H_{2}},
$$

where $w=\left(w_{1}, w_{2}\right), \widetilde{w}=\left(\widetilde{w}_{1}, \widetilde{w}_{2}\right)$.

Consider now a nonlocal b.v.p. associated to a system of differential equations

$$
\left\{\begin{array}{l}
x^{\prime}(t)=f_{1}(t, x(t), y(t)) \text { for a.e. } t \in I, \\
y^{\prime}(t)=f_{2}(t, x(t), y(t)) \text { for a.e. } t \in I, \\
x(0)=M_{1} x \\
y(0)=M_{2} y
\end{array}\right.
$$

where $f_{i}: I \times H_{1} \times H_{2} \rightarrow H_{i}$ and $M_{i}: C\left(I, H_{i}\right) \rightarrow H_{i}(i=1,2)$.

Assume that, for $i=1,2$ :

(h1) the maps $f_{i}$ are (globally) measurable;

( $h 2$ ) for a.e. $t \in I, f_{i}(t, \cdot): H_{1} \times H_{2} \rightarrow H_{i}$ are $E-E_{i}$ continuous;

(h3) for every bounded subset $\Omega \subset H$ there exist $v_{\Omega}^{(i)} \in L_{+}^{1}[0, T]$ such that for each $\omega \in \Omega$ we have

$$
\left\|f_{i}(t, \omega)\right\|_{H_{i}} \leq v_{\Omega}^{(i)}(t)
$$

for a.e. $t \in I$;

(h4) $M_{i}$ are linear bounded maps such that $\left\|M_{1}\right\|^{2}+\left\|M_{2}\right\|^{2} \leq 1$.

By a solution to problem (??) we mean a pair $(x, y)$ consisting of functions $x \in$ $W^{1,1}\left(I, H_{1}\right)$ and $y \in W^{1,1}\left(I, H_{2}\right)$ that satisfy (??).

With $z=(x, y)$, problem (??) can be rewritten as

$$
\left\{\begin{array}{l}
z^{\prime}(t)=f(t, z(t)) \text { for a.e. } t \in I, \\
z(0)=M z
\end{array}\right.
$$

where $f: I \times H \rightarrow H$ and $M: C(I, H) \rightarrow H$ are defined by

$$
f(t, w)=\left(f_{1}\left(t, w_{1}, w_{2}\right), f_{2}\left(t, w_{1}, w_{2}\right)\right), \quad w=\left(w_{1}, w_{2}\right) \in H,
$$

and

$$
M z=\left(M_{1} x, M_{2} y\right), \quad z=(x, y) \in C(I, H) .
$$

By using conditions $(h 1)-(h 4)$, it is easy to verify that the maps $f$ and $M$ satisfy $(f 1)-(f 3)$ and $(M)$, respectively. Applying Theorem ?? we easily obtain the following assertion.

Theorem 7.1. Let conditions $(h 1)-(h 4)$ hold. If there exist $R_{0}>r_{0}>0$ such that

$$
\left\langle w_{1}, f_{1}\left(t, w_{1}, w_{2}\right)\right\rangle_{H_{1}}+\left\langle w_{2}, f_{2}\left(t, w_{1}, w_{2}\right)\right\rangle_{H_{2}}<0,
$$


for all $w=\left(w_{1}, w_{2}\right) \in B_{H}\left(0, r_{0}, R_{0}\right)$ and for a.e. $t \in I$, then problem (??) has a solution.

To illustrate the result let us consider the following system of integro-differential equations

$$
\left\{\begin{array}{l}
\frac{\partial u(t, \xi)}{\partial t}+a \int_{\Omega} u(t, \xi) d \xi+b u(t, \xi)=f_{1}(t, u(t, \xi), v(t, \xi)) \\
\frac{\partial v(t, \xi)}{\partial t}+c v(t, \xi)=f_{2}(t, u(t, \xi), v(t, \xi)) \\
u(0, \xi)=\sum_{1}^{m} \alpha_{j} u\left(t_{j}, \xi\right) \\
v(0, \xi)=\int_{0}^{1} g(t) v(t, \xi) d t
\end{array}\right.
$$

for a.e. $t \in[0,1]$ and for all $\xi \in \Omega$, where $\Omega \subset \mathbb{R}^{k}(k \geq 2)$ is an open bounded domain with Lipschitz boundary; $a, b, c>0 ; \alpha_{j} \in \mathbb{R} ; 0<t_{1}<\cdots<t_{m} \leq 1$; $f_{i}:[0,1] \times \mathbb{R} \times \mathbb{R} \rightarrow \mathbb{R}(i=1,2)$ are continuous maps and $g \in L^{1}[0,1]$.

By a solution to problem (??) we mean a pair $(u, v)$ consisting of continuous functions $u, v:[0,1] \times \Omega \rightarrow \mathbb{R}$ whose partial derivatives $\frac{\partial u(t, \xi)}{\partial t}$ and $\frac{\partial v(t, \xi)}{\partial t}$ exist and satisfy (??). Moreover, we can consider relations (??) as the law of evolution of a feedback control system with the state function $u(t, \xi)$ and the control function $v(t, \xi)$. Our goal can be formulated as the finding of the state and control as continuous functions $u(t, \xi)$ and $v(t, \xi)$ such that at every value $t$ the functions $u(t, \cdot)$ and $v(t, \cdot)$ belong to the Sobolev space $W^{1,2}(\Omega)$.

We assume the following conditions.

$(h 1)^{\prime}$ the partial derivatives $\frac{\partial f_{i}}{\partial z_{j}}:[0,1] \times \mathbb{R} \times \mathbb{R} \rightarrow \mathbb{R}(i, j=1,2)$ are continuous;

$(h 2)^{\prime}$ there exist positive numbers $N_{i, j}(i, j=1,2)$ such that $N_{11}+\frac{N_{12}+N_{21}}{2}<b$ and $N_{22}+\frac{N_{12}+N_{21}}{2}<c$ and

$$
\left|\frac{\partial f_{i}}{\partial z_{j}}\left(t, z_{1}, z_{2}\right)\right| \leq N_{i j}
$$

for all $\left(t, z_{1}, z_{2}\right) \in[0,1] \times \mathbb{R} \times \mathbb{R}$

$(h 3)^{\prime}\left(\sum_{j=1}^{m}\left|\alpha_{j}\right|\right)^{2}+\|g\|_{1}^{2} \leq 1$, where $\|g\|_{1}=\int_{0}^{1}|g(t)| d t$.

Theorem 7.2. Let conditions $(h 1)^{\prime}-(h 3)^{\prime}$ hold. Then problem (??) has a solution.

Proof. Let $H_{1}=W^{1,2}(\Omega)$ and $E_{1}=L^{2}(\Omega)$. From $(h 1)^{\prime}-(h 2)^{\prime}$ it follows that

$$
\left|f_{i}\left(t, z_{1}, z_{2}\right)\right| \leq\left|f_{i}(t, 0,0)\right|+N_{i 1}\left|z_{1}\right|+N_{i 2}\left|z_{2}\right|,
$$

for all $\left(t, z_{1}, z_{2}\right) \in[0,1] \times \mathbb{R} \times \mathbb{R}$ and $i=1,2$.

For each $t \in[0,1]$ set $x(t)=u(t, \cdot)$ and $y(t)=v(t, \cdot)$. Then we can reduce (??) to 
the following problem

$$
\left\{\begin{array}{l}
x^{\prime}(t)=\overline{f_{1}}(t, x(t), y(t)), \text { for a.e. } t \in[0,1], \\
y^{\prime}(t)=\overline{f_{2}}(t, x(t), y(t)), \text { for a.e. } t \in[0,1], \\
x(0)=M_{1} x, \\
y(0)=M_{2} y
\end{array}\right.
$$

where $\overline{f_{i}}:[0,1] \times H_{1} \times H_{1} \rightarrow H_{1}(i=1,2)$ are defined as

$$
\begin{aligned}
& \overline{f_{1}}\left(t, w_{1}, w_{2}\right)(\xi)=-a \int_{\Omega} w_{1}(\tau) d \tau-b w_{1}(\xi)+f_{1}\left(t, w_{1}(\xi), w_{2}(\xi)\right), \\
& \overline{f_{2}}\left(t, w_{1}, w_{2}\right)(\xi)=-c w_{2}(\xi)+f_{2}\left(t, w_{1}(\xi), w_{2}(\xi)\right),
\end{aligned}
$$

and $M_{1}, M_{2}: C\left(I, H_{1}\right) \rightarrow H_{1}$ are given by

$$
M_{1} x=\sum_{j=1}^{m} \alpha_{j} x\left(t_{i}\right), \quad M_{2} y=\int_{0}^{1} g(t) y(t) d t .
$$

It is clear that $M_{1}$ and $M_{2}$ are linear bounded maps with $\left\|M_{1}\right\|^{2}+\left\|M_{2}\right\|^{2} \leq 1$.

Notice that for every $(t, \xi) \in(0,1) \times \Omega$

$$
\begin{aligned}
\frac{D \overline{f_{1}}\left(t, w_{1}, w_{2}\right)(\xi)}{D \xi}=-b D w_{1}(\xi) & +\frac{\partial f_{1}\left(t, w_{1}(\xi), w_{2}(\xi)\right)}{\partial z_{1}} D w_{1}(\xi) \\
& +\frac{\partial f_{1}\left(t, w_{1}(\xi), w_{2}(\xi)\right)}{\partial z_{2}} D w_{2}(\xi),
\end{aligned}
$$

and

$$
\begin{aligned}
\frac{D \overline{f_{2}}\left(t, w_{1}, w_{2}\right)(\xi)}{D \xi}=-c D w_{2}(\xi) & +\frac{\partial f_{2}\left(t, w_{1}(\xi), w_{2}(\xi)\right)}{\partial z_{1}} D w_{1}(\xi) \\
& +\frac{\partial f_{2}\left(t, w_{1}(\xi), w_{2}(\xi)\right)}{\partial z_{2}} D w_{2}(\xi),
\end{aligned}
$$

From $(h 1)^{\prime}$ it follows that the maps $\overline{f_{1}}$ and $\overline{f_{2}}$ are well-defined.

Let $H=H_{1} \times H_{1}, E=E_{1} \times E_{1}$ and define the maps

$$
\begin{gathered}
f:[0,1] \times H \rightarrow H, \\
f(t, w)=\left(\overline{f_{1}}\left(t, w_{1}, w_{2}\right), \overline{f_{2}}\left(t, w_{1}, w_{2}\right)\right), w=\left(w_{1}, w_{2}\right) \in H,
\end{gathered}
$$

and

$$
M: C(I, H) \rightarrow H, M z=\left(M_{1} x, M_{2} y\right),
$$

for all $z=(x, y) \in C(I, H) ; x, y \in C\left(I, H_{1}\right)$.

Following the lines of the proof of Theorem ??, we obtain that the maps $\bar{f}_{1}$ and $\bar{f}_{2}$ satisfy conditions $(h 1)-(h 3)$.

Now for $w=\left(w_{1}, w_{2}\right) \in H$ and for $t \in[0,1]$ we have

$$
\langle w, f(t, w)\rangle_{H}=\left\langle w_{1}, \overline{f_{1}}\left(t, w_{1}, w_{2}\right)\right\rangle_{H_{1}}+\left\langle w_{2}, \overline{f_{2}}\left(t, w_{1}, w_{2}\right)\right\rangle_{H_{1}} .
$$


On the other hand,

$$
\begin{aligned}
& \left\langle w_{1}, \overline{f_{1}}\left(t, w_{1}, w_{2}\right)\right\rangle_{H_{1}}= \\
& =-a\left(\int_{\Omega} w_{1}(\xi) d \xi\right)^{2}-b\left\|w_{1}\right\|_{H_{1}}^{2}+\int_{\Omega} f_{1}\left(t, w_{1}(\xi), w_{2}(\xi)\right) w_{1}(\xi) d \xi \\
& +\int_{\Omega} \frac{\partial f_{1}\left(t, w_{1}(\xi), w_{2}(\xi)\right)}{\partial z_{1}}\left|D w_{1}(\xi)\right|^{2} d \xi \\
& +\int_{\Omega} \frac{\partial f_{1}\left(t, w_{1}(\xi), w_{2}(\xi)\right)}{\partial z_{2}} D w_{1}(\xi) D w_{2}(\xi) d \xi \\
& \leq-b\left\|w_{1}\right\|_{H_{1}}^{2}+\int_{\Omega}\left|w_{1}(\xi)\right|\left(\left|f_{1}(t, 0,0)\right|+N_{11}\left|w_{1}(\xi)\right|+N_{12}\left|w_{2}(\xi)\right|\right) d \xi \\
& +N_{11} \int_{\Omega}\left|D w_{1}(\xi)\right|^{2} d \xi+N_{12} \int_{\Omega}\left|D w_{1}(\xi) D w_{2}(\xi)\right| d \xi \\
& \leq\left(-b+N_{11}\right)\left\|w_{1}\right\|_{H_{1}}^{2}+\beta_{1} \int_{\Omega}\left|w_{1}(\xi)\right| d \xi+N_{12} \int_{\Omega}\left|w_{1}(\xi)\right|\left|w_{2}(\xi)\right| d \xi \\
& +N_{12} \int_{\Omega}\left|D w_{1}(\xi) D w_{2}(\xi)\right| d \xi \\
& \leq\left(-b+N_{11}\right)\left\|w_{1}\right\|_{H_{1}}^{2}+\beta_{1}|\Omega|\left\|w_{1}\right\|_{H_{1}}+N_{12}\left\|w_{1}\right\|_{H_{1}}\left\|w_{2}\right\|_{H_{1}},
\end{aligned}
$$

where $\beta_{1}=\max _{[0,1]}\left|f_{1}(t, 0,0)\right|$.

$$
\begin{aligned}
& \begin{array}{l}
\left\langle w_{2}, \overline{f_{2}}\left(t, w_{1}, w_{2}\right)\right\rangle_{H_{1}}= \\
=-c\left\|w_{2}\right\|_{H_{1}}^{2}+\int_{\Omega} f_{2}\left(t, w_{1}(\xi), w_{2}(\xi)\right) w_{2}(\xi) d \xi \\
\quad+\int_{\Omega} \frac{\partial f_{2}\left(t, w_{1}(\xi), w_{2}(\xi)\right)}{\partial z_{2}}\left|D w_{2}(\xi)\right|^{2} d \xi \\
\quad+\int_{\Omega} \frac{\partial f_{2}\left(t, w_{1}(\xi), w_{2}(\xi)\right)}{\partial z_{1}} D w_{1}(\xi) D w_{2}(\xi) d \xi \\
\leq-c\left\|w_{2}\right\|_{H_{1}}^{2}+\int_{\Omega}\left|w_{2}(\xi)\right|\left(\left|f_{2}(t, 0,0)\right|+N_{21}\left|w_{1}(\xi)\right|+N_{22}\left|w_{2}(\xi)\right|\right) d \xi \\
\quad+N_{22} \int_{\Omega}\left|D w_{2}(s)\right|^{2} d \xi+N_{21} \int_{\Omega}\left|D w_{1}(\xi) D w_{2}(\xi)\right| d \xi \\
\leq\left(-c+N_{22}\right)\left\|w_{2}\right\|_{H_{1}}^{2}+\beta_{2} \int_{\Omega}\left|w_{2}(\xi)\right| d \xi+N_{21} \int_{\Omega}\left|w_{1}(\xi)\right|\left|w_{2}(\xi)\right| d \xi \\
\quad+N_{21} \int_{\Omega}\left|D w_{1}(\xi) D w_{2}(\xi)\right| d \xi
\end{array} \\
& \leq\left(-c+N_{22}\right)\left\|w_{2}\right\|_{H_{1}}^{2}+\beta_{2}|\Omega|\left\|w_{2}\right\|_{H_{1}}+N_{21}\left\|w_{1}\right\|_{H_{1}}\left\|w_{2}\right\|_{H_{1}},
\end{aligned}
$$

Set

$$
R=\min \left\{b-N_{11}-\frac{N_{12}+N_{21}}{2}, c-N_{22}-\frac{N_{12}+N_{21}}{2}\right\} .
$$


Then

$$
\begin{gathered}
\langle w, f(t, w)\rangle_{H} \leq-\left(b-N_{11}\right)\left\|w_{1}\right\|_{H_{1}}^{2}-\left(c-N_{22}\right)\left\|w_{2}\right\|_{H_{1}}^{2} \\
+|\Omega|\left(\beta_{1}\left\|w_{1}\right\|_{H_{1}}+\beta_{2}\left\|w_{2}\right\|_{H_{1}}\right)+\left(N_{12}+N_{21}\right)\left\|w_{1}\right\|_{H_{1}}\left\|w_{2}\right\|_{H_{1}} \\
\leq-\left(b-N_{11}-\frac{N_{12}+N_{21}}{2}\right)\left\|w_{1}\right\|_{H_{1}}^{2}-\left(c-N_{22}-\frac{N_{12}+N_{21}}{2}\right)\left\|w_{2}\right\|_{H_{1}}^{2} \\
\quad+|\Omega|\left(\beta_{1}\left\|w_{1}\right\|_{H_{1}}+\beta_{2}\left\|w_{2}\right\|_{H_{1}}\right) \\
\leq-R\left(\left\|w_{1}\right\|_{H_{1}}^{2}+\left\|w_{2}\right\|_{H_{1}}^{2}\right)+|\Omega|\left(\beta_{1}\left\|w_{1}\right\|_{H_{1}}+\beta_{2}\left\|w_{2}\right\|_{H_{1}}\right) \\
\leq-R\|w\|_{H}^{2}+|\Omega| \sqrt{\beta_{1}^{2}+\beta_{2}^{2}} \cdot\|w\|_{H}<0,
\end{gathered}
$$

provided

$$
\|w\|_{H}>\frac{|\Omega| \sqrt{\beta_{1}^{2}+\beta_{2}^{2}}}{R} .
$$

Applying Theorem ?? we obtain the existence of a solution to (??), and so, we conclude that problem (??) has a solution.

\section{Uniqueness results}

We now examine the uniqueness of the solutions for the problems seen in the previous sections. To this aim we need to consider stronger $H$-regularity assumptions on the term $f$, however we are able to weaken the assumption $(f 1)$. Precisely, we introduce the following condition

$\left(f 1^{\prime}\right) \quad$ (i) $f(\cdot, x)$ is measurable for all $x \in H$;

(ii) there exists a function $\eta \in L_{+}^{1}[0, T]$ such that

$$
\left\|f\left(t, \omega_{1}\right)-f\left(t, \omega_{2}\right)\right\|_{H} \leq \eta(t)\left\|\omega_{1}-\omega_{2}\right\|_{H}
$$

for each $\omega_{1}, \omega_{2} \in \overline{B_{H}\left(0, R_{0}\right)}$ and for a.e. $t \in I$, with $R_{0}$ as in $(f 4)$.

Notice that $\left(f 1^{\prime}\right)$ implies that $f$ is a Carathéodory function; hence it is globally measurable, i.e. $(f 1)$ is satisfied. The following result deals with problem (1.1)-(1.2).

Theorem 8.1. Let conditions $\left(f 1^{\prime}\right),(f 2),(f 4)$ and $(M)$ hold and assume that $\|M\| e^{\|\eta\|_{1}}<1$.

Then problem (1.1)-(1.2) admits a unique solution with values in $\overline{B_{H}\left(0, R_{0}\right)}$.

Proof. The existence follows by Theorem ??. Assume by contradiction the existence of two solutions $y_{1}, y_{2}$ to problem (??)-(??) in $\overline{B_{H}\left(0, R_{0}\right)}$. We have that

$$
\begin{aligned}
& y_{1}(t)=M y_{1}+\int_{0}^{t} f\left(s, y_{1}(s)\right) d s, \\
& y_{2}(t)=M y_{2}+\int_{0}^{t} f\left(s, y_{2}(s)\right) d s .
\end{aligned}
$$


Hence for any $t \in I$,

$$
\begin{aligned}
& \left\|y_{1}(t)-y_{2}(t)\right\|_{H}=\left\|M\left(y_{1}-y_{2}\right)+\int_{0}^{t}\left(f\left(s, y_{1}(s)\right)-f\left(s, y_{2}(s)\right)\right) d s\right\|_{H} \leq \\
& \|M\|\left\|y_{1}-y_{2}\right\|_{C}+\int_{0}^{t}\left\|f\left(s, y_{1}(s)\right)-f\left(s, y_{2}(s)\right)\right\|_{H} d s \leq \\
& \|M\|\left\|y_{1}-y_{2}\right\|_{C}+\int_{0}^{t} \eta(s)\left\|y_{1}(s)-y_{2}(s)\right\|_{H} d s .
\end{aligned}
$$

Let $L=\left\|y_{1}-y_{2}\right\|_{C}$, applying the Gronwall Bellmann inequality we obtain

$$
\left\|y_{1}(t)-y_{2}(t)\right\|_{H} \leq\|M\| L\left(\exp \left\{\int_{0}^{t} \eta(s) d s\right\}\right) .
$$

Hence

$$
L=\sup _{0 \leq t \leq T}\left\|y_{1}(t)-y_{2}(t)\right\|_{H} \leq\|M\| L e^{\|\eta\|_{1}}<L,
$$

obtaining the contradiction.

Let us consider now the semilienar Problem (??). As in Section ??, we introduce the function $\widetilde{f}(t, x):=-A x+f(t, x),(t, x) \in[0, T] \times H$. If $f$ satisfies $\left(f 1^{\prime}\right)($ ii), it is easy to see that

$$
\left\|\tilde{f}\left(t, \omega_{1}\right)-\widetilde{f}\left(t, \omega_{2}\right)\right\|_{H} \leq(\|A\|+\eta(t))\left\|\omega_{1}-\omega_{2}\right\|_{H} .
$$

Hence, by Theorem ??, under condition $\left(f 1^{\prime}\right),(f 2),(f 4),(M)$ and assuming $\|M\| e^{\|A\| T} e^{\|\eta\|_{1}}<1$, Problem (??) admits a unique solution.

Moreover, under the same hypotheses $\left(f 1^{\prime}\right),(f 2),(f 4)$ and $(M)$ it is possible to prove the existence of a unique solution in $\overline{B_{H}\left(0, R_{0}\right)}$ for Problem (??) too. Indeed, assume by contradiction the existence of two solutions $x^{1}, x^{2}$ of Problem (??) in $\overline{B_{H}\left(0, R_{0}\right)}$. Define

$$
x_{n}^{1}(t)=\left\{\begin{array}{l}
x^{1}(t) \text { for } t \in[0, n] \\
x^{1}(n) \text { for } t \geq n
\end{array}\right.
$$

and

$$
x_{n}^{2}(t)=\left\{\begin{array}{l}
x^{2}(t) \text { for } t \in[0, n], \\
x^{2}(n) \text { for } t \geq n .
\end{array}\right.
$$

Hence, we have that for every $n \in \mathbb{N}$ the functions $x_{n}^{1}, x_{n}^{2}$ are solutions of the problem

$$
\left\{\begin{array}{l}
x^{\prime}(t)=f(t, x(t)) \text { for a.e. } t \in[0, n], \\
x(0)=0 .
\end{array}\right.
$$

in $\overline{B_{H}\left(0, R_{0}\right)}$. This is in contradiction with Theorem ?? 
Finally, as in Section ??, let $H_{i}(i=1,2)$ be separable Hilbert spaces which are compactly embedded in Banach spaces $E_{i}$, respectively. Consider the product spaces $H=H_{1} \times H_{2}, E=E_{1} \times E_{2}$ and consider $f_{i}: I \times H_{1} \times H_{2} \rightarrow H_{i}$ and $M_{i}: C\left(I, H_{i}\right) \rightarrow H_{i}(i=1,2)$. Assume that

$\left(f 1^{\prime \prime}\right) \quad$ (i) $f_{i}(\cdot, x)$ is measurable for all $x \in H,(i=1,2)$;

(ii) there exist two functions $\eta_{1}, \eta_{2} \in L_{+}^{1}[0, T]$ such that

$$
\left\|f_{i}\left(t, \omega_{1}\right)-f_{i}\left(t, \omega_{2}\right)\right\|_{H_{i}} \leq \eta_{i}(t)\left\|\omega_{1}-\omega_{2}\right\|_{H}(i=1,2),
$$

for each $\omega_{1}, \omega_{2} \in \overline{B_{H}\left(0, R_{0}\right)}$ and for a.e. $t \in I$, with $R_{0}$ as in $(f 4)$.

Under the conditions $\left(f 1^{\prime \prime}\right),(h 2),(h 4)$, and $\|M\| e^{\|\gamma\|_{1}}<1$, with $\gamma(t)=$ $\sqrt{\left(\eta_{1}(t)\right)^{2}+\left(\eta_{2}(t)\right)^{2}}, t \in I$ it is easy to prove that the nonlocal b.v.p. associated to the system of differential equations (??) has a unique solution.

\section{Non-smooth bounding function in a Hilbert space}

Let $H$ be a separable Hilbert space which is compactly embedded in a Banach space $E$. We consider again problem (??)-(??):

$$
\left\{\begin{array}{l}
x^{\prime}(t)=f(t, x(t)), \text { for a.e. } t \in I, \\
x(0)=M x,
\end{array}\right.
$$

where $f: I \times H \rightarrow H$ satisfies conditions $(f 1)-(f 3)$ and $M: C(I, H) \rightarrow H$ is a bounded linear map.

Let $U \subset H$ be an open subset. A function $V: U \rightarrow \mathbb{R}$ is said to be Lipschitzian with constant $L>0$ if

$$
|V(x)-V(y)| \leq L\|x-y\|_{H} \text { for all } x, y \in U .
$$

A function $V$ is said to be locally Lipschitzian if for every $x \in U$ there exists $\varepsilon>0$ such that $B_{H}(x, \varepsilon) \subset U$ and the restriction $V_{\left.\right|_{B_{H}(x, \varepsilon)}}$ is Lipschitzian. It is easy to see that if $V$ is locally Lipschitzian, then for every $x \in U$ and for all $w \in H$ the following limit

$$
\liminf _{h \rightarrow 0^{-}} \frac{V(x+h w)-V(x)}{h}
$$

exists and is finite.

Definition 9.1. A locally Lipschitzian functional $V: H \rightarrow \mathbb{R}$ is said to be a bounding function for equation (??), if there exist $\varepsilon>0$ and an open bounded convex subset $K \subset H$ such that

(V1) $V_{\left.\right|_{\partial K}}=0$ and $V(w) \leq 0$ for all $w \in O_{\varepsilon}^{K}(\partial K)=K \cap O_{\varepsilon}(\partial K)$, where $O_{\varepsilon}(\partial K)$ denotes the $\varepsilon-$ neighborhood of $\partial K$ in $H$; 
$(V 2)$ for a.e. $t \in I$ the relation

$$
\liminf _{n \rightarrow \infty} \operatorname{sign}\left(\liminf _{h \rightarrow 0^{-}} \frac{V\left(w+h P_{n} f(t, w)\right)-V(w)}{h}\right)=-1
$$

holds true for all $w \in O_{\varepsilon}^{K_{n}}\left(\partial K_{n}\right)$, where $K_{n}=K \cap H_{n}$; or equivalently,

$$
\liminf _{n \rightarrow \infty} \operatorname{sign}\left(\liminf _{h \rightarrow 0^{-}} \frac{V\left(w+\lambda h P_{n} f(t, w)\right)-V(w)}{h}\right)=-1
$$

holds true for all $(\lambda, w) \in(0,1) \times O_{\varepsilon}^{K_{n}}\left(\partial K_{n}\right)$, where $K_{n}=K \cap H_{n}$.

Condition $(V 2)$ means that there exists a subsequence of spaces $\left\{H_{n_{m}}\right\}$ such that for all $n_{m}$ the relation

$$
\liminf _{h \rightarrow 0^{-}} \frac{V\left(w+h P_{n_{m}} f(t, w)\right)-V(w)}{h}<0
$$

holds true for all $w \in O_{\varepsilon}^{K_{n_{m}}}\left(\partial K_{n_{m}}\right)$.

Notice that if $V \in C^{1}(H, \mathbb{R})$, i.e. $V$ is continuously differentiable, then for every $w \in H$ the Frétcher derivative $\nabla V(w)$ of $V$ at $w$ can be identified with an element in $H$. Hence, for a.e. $t \in I$ and for every $n \in \mathbb{N}$ :

$$
\liminf _{h \rightarrow 0^{-}} \frac{V\left(w+h P_{n} f(t, w)\right)-V(w)}{h}=\left\langle\nabla V(w), P_{n} f(t, w)\right\rangle_{H}
$$

for each $w \in O_{\varepsilon}^{K_{n}}\left(\partial K_{n}\right)$.

Therefore, in case $V \in C^{1}(I, H)$, condition $(V 2)$ can be written as

$(V 2)^{\prime}$ there exists a subsequence of spaces $\left\{H_{n_{m}}\right\}$ such that

$$
\left\langle\nabla V(w), P_{n_{m}} f(t, w)\right\rangle_{H}<0 \text { for a.e. } t \in I
$$

and for each $w \in O_{\varepsilon}^{K_{n_{m}}}\left(\partial K_{n_{m}}\right)$.

Moreover, if $\nabla V$ is projectively homogeneous, i.e., there exists $n_{0} \in \mathbb{N}$ such that

$$
P_{n} \nabla V(w)=\nabla V\left(P_{n} w\right)
$$

for all $n \geq n_{0}$ and all $w \in H$, then for every $n \geq n_{0}$ we have

$$
\begin{aligned}
\left\langle\nabla V(w), P_{n} f(t, w)\right\rangle_{H} & =\left\langle P_{n} \nabla V(w), f(t, w)\right\rangle_{H} \\
& =\langle\nabla V(w), f(t, w)\rangle_{H} \text { for a.e. } t \in I
\end{aligned}
$$

and for each $w \in H_{n}$.

Therefore, in case $V \in C^{1}(H, \mathbb{R})$ and $\nabla V$ is projectively homogeneous, condition $(V 2)$ can be replaced with the following condition: 
$(V 2)^{\prime \prime}$ the relation

$$
\langle\nabla V(w), f(t, w)\rangle_{H}<0 \text { for a.e. } t \in I
$$

holds true for each $w \in O_{\varepsilon}^{K}(\partial K)$.

Notice that a trivial example of projectively homogenous potential $\nabla V(w)=$ $w, w \in H$, was used in Section 4 to obtain our main result.

Since for every bounding function $V$ of equation (??) there exist an open bounded convex set $K$ and a number $\varepsilon>0$ such that $(V 1)-(V 2)$ are satisfied, in the sequel (for short) we will call $V$ a $(K, \varepsilon)$-bounding function.

The following assertion illustrates the geometric sense of a bounding function.

Lemma 9.1. Let $V$ be a $(K, \varepsilon)$-bounding function to equation (??). Then there exists a subsequence of spaces $\left\{H_{n_{m}}\right\}$ such that if the solution operator

$$
\mathcal{T}_{n_{m}}: C\left(I, \overline{K_{n_{m}}}\right) \times(0,1) \rightarrow C\left(I, H_{n_{m}}\right)
$$

of the linearized problem

$$
\left\{\begin{array}{l}
x^{\prime}(t)=\lambda P_{n_{m}} f(t, y(t)), \text { for a.e. } t \in I, \\
x(0)=\lambda P_{n_{m}} M y
\end{array}\right.
$$

has a fixed point $x_{n_{m}}$, i.e. $x_{n_{m}}=\mathcal{T}_{n_{m}}\left(x_{n_{m}}, \lambda\right)$ for some $\lambda \in(0,1)$, such that $x_{n_{m}}(0) \notin \partial K_{n_{m}}$, then $x_{n_{m}}(t) \in K_{n_{m}}$ for all $t \in I$.

Proof. From the definition of a bounding function it follows that there exists a subsequence of spaces $\left\{H_{n_{m}}\right\}$ such that for a.e. $t \in I$ we have

$$
\liminf _{h \rightarrow 0^{-}} \frac{V\left(w+h P_{n_{m}} f(t, w)\right)-V(w)}{h}<0 \text { for each } w \in O_{\varepsilon}^{K_{n_{m}}}\left(\partial K_{n_{m}}\right)
$$

or equivalently,

$$
\liminf _{h \rightarrow 0^{-}} \frac{V\left(w+\lambda h P_{n_{m}} f(t, w)\right)-V(w)}{h}<0
$$

for each $(\lambda, w) \in(0,1) \times O_{\varepsilon}^{K_{n_{m}}}\left(\partial K_{n_{m}}\right)$.

Now, assume that the fixed point $x_{n_{m}}$ touches the boundary $\partial K_{n_{m}}$. Since $x_{n_{m}}(0) \notin \partial K_{n_{m}}$ we can choose $t_{0} \in(0, T]$ such that $x_{n_{m}}\left(t_{0}\right) \in \partial K_{n_{m}}$ and $x_{n_{m}}(t) \in K_{n_{m}}$ for sufficiently small $t<t_{0}$. From the locally Lipschitz property of $V$ it follows that there exists $\delta>0$ such that the restriction of $V$ on $B_{H}\left(x_{n_{m}}\left(t_{0}\right), \delta\right)$ is Lipschitzian with constant $L>0$. We can choose $\delta \in(0, \varepsilon)$ such that $x_{n_{m}}(t) \in B_{H}\left(x_{n_{m}}\left(t_{0}\right), \delta\right) \cap K_{n_{m}}$ for all $t \in\left(t_{0}-\delta, t_{0}\right)$. It is easy to see that the function $g_{n_{m}}(t)=V\left(x_{n_{m}}(t)\right)$ is absolutely continuous in $t \in\left(t_{0}-\delta, t_{0}\right)$, and so $g_{n_{m}}^{\prime}(t)$ exists for a.e. $t \in\left(t_{0}-\delta, t_{0}\right)$. Hence,

$$
\int_{t_{0}-\delta}^{t_{0}} g_{n_{m}}^{\prime}(s) d s=V\left(x_{n_{m}}\left(t_{0}\right)\right)-V\left(x_{n_{m}}\left(t_{0}-\delta\right)\right)=-V\left(x_{n_{m}}\left(t_{0}-\delta\right)\right) \geq 0 .
$$


On the other hand, since $x_{n_{m}}=\mathcal{T}_{n_{m}}\left(x_{n_{m}}, \lambda\right)$ we have

$$
x_{n_{m}}^{\prime}(t)=\lambda P_{n_{m}} f\left(t, x_{n_{m}}(t)\right) \text {, for a.e. } t \in\left(t_{0}-\delta, t_{0}\right) .
$$

For a.e. $t \in\left(t_{0}-\delta, t_{0}\right)$ and for a sufficiently small $h<0$ take

$$
\varphi(h):=x_{n_{m}}(t+h)-x_{n_{m}}(t)-x_{n_{m}}^{\prime}(t) h
$$

and

$$
\Delta(h):=\frac{V\left(x_{n_{m}}(t)+x_{n_{m}}^{\prime}(t) h+\varphi(h)\right)-V\left(x_{n_{m}}(t)+x_{n_{m}}^{\prime}(t) h\right)}{h} .
$$

According to the Lipschitz property of $V$ and the definition of $\varphi(h)$ we have

$$
|\Delta(h)| \leq \frac{L\|\varphi(h)\|_{H}}{|h|} \rightarrow 0 \text { as } h \rightarrow 0 .
$$

Therefore, since

$$
\frac{g_{n_{m}}(t+h)-g_{n_{m}}(t)}{h}=\frac{V\left(x_{n_{m}}(t)+x_{n_{m}}^{\prime}(t) h\right)-V\left(x_{n_{m}}(t)\right)}{h}+\Delta(h),
$$

we obtain

$$
\liminf _{h \rightarrow 0^{-}} \frac{g_{n_{m}}(t+h)-g_{n_{m}}(t)}{h}=\liminf _{h \rightarrow 0^{-}} \frac{V\left(x_{n_{m}}(t)+x_{n_{m}}^{\prime}(t) h\right)-V\left(x_{n_{m}}(t)\right)}{h}<0 .
$$

Consequently, $\int_{t_{0}-\delta}^{t_{0}} g_{n_{m}}^{\prime}(s) d s<0$, giving the contradiction.

Assume that the operator $M$ satisfies the following condition:

$(M)^{\prime}$ For every sufficiently large $n \in \mathbb{N}$, if $x_{n}$ is a fixed point of the solution map $\mathcal{T}_{n}: C\left(I, \overline{K_{n}}\right) \times(0,1) \rightarrow C\left(I, H_{n}\right)$ of the linearized problem

$$
\left\{\begin{array}{l}
x^{\prime}(t)=\lambda P_{n} f(t, y(t)) \text { for a.e. } t \in I, \\
x(0)=\lambda P_{n} M y
\end{array}\right.
$$

then $x_{n}(0) \notin \partial K_{n}$.

Let us consider some sufficient conditions that provide condition $(M)^{\prime}$.

Proposition 9.1. The operator $M$ satisfies condition $(M)^{\prime}$ if the set $K$ contains 0 and at least one of the following assumptions is fulfilled.

(i) $M(Q) \subseteq \bar{K}$, where $Q=C(I, \bar{K})$;

(ii) $K$ is an open ball $B_{H}(0, r)$ (for some $r>0$ ) and $\|M\| \leq 1$.

Proof. Let assumption $(i)$ holds. Assume that $x_{n}=\mathcal{T}_{n}\left(x_{n}, \lambda\right)$ and $x_{n}(0) \in \partial K_{n}$. Therefore, $\lambda P_{n} M x_{n} \in \partial K_{n}$. Since $M x_{n} \in \bar{K}$ we have $P_{n} M x_{n} \in \overline{K_{n}}$. From the convexity of the set $K$ and the assumption that $0 \in K$ it follows that $\lambda P_{n} M x_{n}=x_{n}(0) \in K_{n}$, for $\lambda \in(0,1)$. That is the contradiction. 
Let assumption (ii) hold and assume again that $x_{n}=\mathcal{T}_{n}\left(x_{n}, \lambda\right)$ and $x_{n}(0) \in$ $\partial K_{n}$. Then

$$
r=\left\|x_{n}(0)\right\|_{H}=\lambda\left\|P_{n} M x_{n}\right\|_{H}<r,
$$

giving the contradiction.

Theorem 9.1. Let conditions $(f 1)-(f 3)$ and $(M)^{\prime}$ hold. If there exists a $(K, \varepsilon)$-bounding function $V$ to equation (??) such that the set $K$ contains 0 , then problem (??)-(??) has a solution with values in $\bar{K}$.

Proof. Set $Q=C(I, \bar{K})$ and

$$
Q^{\left(n_{m}\right)}=Q \cap C\left(I, H_{n_{m}}\right)=\left\{x \in C\left(I, H_{n_{m}}\right): x(t) \in \overline{K_{n_{m}}}, \forall t \in I\right\},
$$

where $\left\{H_{n_{m}}\right\}$ is the subsequence of spaces from Lemma ??.

Consider the map $\mathcal{T}_{n_{m}}: Q^{\left(n_{m}\right)} \times[0,1] \rightarrow C\left(I, H_{n_{m}}\right)$, where $\mathcal{T}_{n_{m}}(y, \lambda)$ is the unique solution of the linearized problem

$$
\left\{\begin{array}{l}
x^{\prime}(t)=\lambda P_{n_{m}} f(t, y(t)) \text { for a.e. } t \in I, \\
x(0)=\lambda P_{n_{m}} M y
\end{array}\right.
$$

Reasoning as in Step 2 of the proof of Theorem ??, we conclude that the map $\mathcal{T}_{n_{m}}$ is completely continuous and

$$
\mathcal{T}_{n_{m}}\left(Q^{\left(n_{m}\right)} \times\{0\}\right)=\{0\} .
$$

By virtue of Lemma ?? the map $\mathcal{T}_{n_{m}}$ has no fixed points on $\partial Q^{\left(n_{m}\right)} \times(0,1)$. Therefore, we again obtain that there exists $y_{n_{m}} \in Q^{\left(n_{m}\right)}$ such that

$$
y_{n_{m}}=\mathcal{T}_{n_{m}}\left(y_{n_{m}}, 1\right)
$$

Analogously to Step 3 in the proof of Theorem ??, we obtain that problem (??)-(??) has a solution whose values are contained in $\bar{K}$.

\section{Acknowledgements}

Supported by the Italian Research Project PRIN 2009 n.2009WEA3T2 Ordinary Differential Equations and Applications, by the Italian Research Project GNAMPA 2013 Topological Methods for Nonlinear Differential Problems and Applications, by a GNAMPA grant for visiting of young researchers and by the Vietnam National Foundation for Science and Technology Development (NAFOSTED) under grant number 101.02-2013.22. 
Benedetti, Loi, Malaguti and Obukhovskii

\section{References}

[1] J. Andres, L. Malaguti, V. Taddei, On boundary values problems in Banach spaces, Dyn. Sys. Appl., 18 (2009): 275-302.

[2] A. I. Alonso, C. Núñez, R. Obaya, Complete guiding sets for a class of almost-periodic differential equations. J. Differential Equations 208(2005), 124-146.

[3] V. Barbu, Nonlinear Semigroups and Differential Equations in Banach Spaces. Noordhoff International Publishing, Leyden, 1976.

[4] I. Benedetti, L. Malaguti, V. Taddei, Semilinear differential inclusions via weak topologies. J. Math. Anal. Appl., 368 (2010), 90-102.

[5] I. Benedetti, V. Taddei, M. Väth, Evolution problems with nonlinear nonlocal boundary conditions, Journal of Dynamics and Differential Equations, 25 (2013), n. 2, 477-503.

[6] I. Benedetti, M. Väth, Semilinear Inclusions with Nonlocal Conditions without Compactness in Non-Reflexive Spaces, submitted.

[7] Yu.G. Borisovich, B.D. Gelman, A.D. Myshkis, V.V. Obukhovskii, Introduction to the Theory of Multivalued Maps and Differential inclusions. Second edition, Librokom, Moscow 2011 (in Russian).

[8] S. Bochner, A.E. Taylor, Linear functionals on certain spaces of abstractly-valued functions. Ann. Math. 39 (4) (1938), 913-944.

[9] F.E. Browder Existence of Periodic Solutions for Nonlinear Equations of Evolution, Proc. Nat. Acad. Sci. U.S.A. 53 (1965) 1100-1103.

[10] A. Capietto, F. Zanolin, A continuation theorem for the periodic bvp in flow-invariant ENRs with applications. J. Differential Equations, 83 (1990), 244-276.

[11] F.H. Clarke, Optimization and Nonsmooth Analysis. Second edition. Classics in Applied Mathematics 5. Society for Industrial and Applied Mathematics (SIAM), Philadelphia, PA, 1990.

[12] Z. Denkowski, S. Migórski, N.S. Papageorgiou, An Introduction to Nonlinear Analysis: Theory. Kluwer Academic Publishers, Boston, MA, 2003.

[13] J. Diestel, J.J., Jr. Uhl, Vector measures. With a foreword by B. J. Pettis. Mathematical Surveys, No. 15. American Mathematical Society, Providence, R.I., 1977.

[14] I. Ekland, R. Temam. Convex Analysis and Variation Problems, North Holland, Amsterdam, 1979.

[15] A. Fonda, Guiding functions and periodic solutions to functional differential equations, Proc. Amer. Math. Soc. 99 (1987), no. 1, 79-85.

[16] D. Gabor, W. Kryszewski, A global bifurcation index for set-valued perturbations of Fredholm operators. Nonlinear Anal. TMA: 73(2010), 2714-2736.

[17] R.E. Gaines, J.L. Mawhin, Ordinary differential equations with nonlinear boundary conditions. J. Differential Equations, 26(1977), 200-222.

[18] R.E. Gaines, J.L. Mawhin, Coincidence Degree and Nonlinear Differential Equations. Lecture Notes in Mathematics, no. 568, Springer-Verlag, Berlin-New York, 1977.

[19] L. Górniewicz, Topological Fixed Point Theory of Multivalued Mappings. 2nd edition. Topological Fixed Point Theory and Its Applications, 4. Springer, Dordrecht, 2006.

[20] L. Górniewicz, S. Plaskacz, Periodic solutions of differential inclusions in $\mathbb{R}^{n}$. Boll. UMI 7-A (1993), 409-420.

[21] M. A. Krasnosel'skii, A. I. Perov, On a certain priciple of existence of bounded, periodic and almost periodic solutions of systems of ordinary differential equations, Dokl. Akad. Nauk SSSR 123 (1958), no. 2, 235-238 (in Russian).

[22] M.A. Krasnosel'skii, The Operator of Translation Along the Trajectories of Differential Equations. Nauka, Moscow 1966 (in Russian); English translation: Translations of Mathematical Monographs 19, Amer. Math. Soc., Providence, R.I. 1968.

[23] M. A. Krasnosel'skii, P. P. Zabreiko, Geometrical Methods of Nonlinear Analysis. 
Nauka, Moscow 1975; English translation: Grundlehren der Mathematischen Wissenschaften 263, Springer-Verlag, Berlin, 1984.

[24] W. Kryszewski, Properties of Set-Valued Mappings. Univ. N. Copernicus Publishing, Torun, 1997.

[25] Lefschetz, Existence of periodic solutions of certain differential equations, Proc. Nat. Acad. Sci. USA 29 (1943), 29-32.

[26] J. Leray et J. Schauder, Topologie et équations fonctionnelles, Ann. Sci. Ecole Norm. Sup. (3) 51 (1934), 45-78.

[27] M. Lewicka, Locally lipschitzian guiding function methods for ODEs. Nonlinear Anal.: TMA. 33(1998), 747-758.

[28] N. V. Loi, Method of guiding functions for differential inclusions in a Hilbert space, Differ. Uravn. 46 (2010), no. 10, 1433-1443 (in Russian); English tranl.: Differ. Equat. 46 (2010), no. 10, 1438-1447.

[29] N. V. Loi, Guiding functions and global bifurcation of periodic solutions of functional differential inclusions with infinite delay. Topol. Meth. Nolinear Anal. 40(2012), 359370.

[30] N.V. Loi, V. Obukhovskii, Guiding functions and generalized periodic problems and applications. Appl. Math. Comp. 218(2012), 11719-11726.

[31] N.V. Loi, V. Obukhovskii, P. Zecca, Non-smooth guiding functions and periodic solutions of functional differential inclusions with infinite delay in Hilbert spaces, Fixed Point Theory, 13(2) (2012), 565-582.

[32] L. Malaguti, P. Rubbioni, Nonsmooth feedback controls of nonlocal dispersal models, submitted.

[33] R. Martin, Nonlinear Operators and Differential Equations in Banach Spaces. Wiley, New York 1976.

[34] J. Mawhin, Periodic solutions of nonlinear functional differential equations. J. Differential Equations, 10(1971), 240-261.

[35] J. Mawhin, Equivalence theorems for nonlinear operator equations and coincidence degree theory for some mappings in locally convex topological vector spaces. J. Differential Equations, 12(1972), 610-636.

[36] J. Mawhin, Boundary value problems for nonlinear second-order vector differential equations. J. Differential Equations, 16(1974), 257-269.

[37] J.Mawhin, Bound sets and Floquet boundary value problems for nonlinear differential equations, Univ. Iagell. Acta Math. 36 (1998) 41-53.

[38] J.Mawhin, H.B. Thompson, Periodic or bounded solutions of Caratheodory systems of ordinary differential equations, J. Dynam. Differential Equations 15 (2003) 327-334.

[39] J. Mawhin, J.R. Ward, Guiding-like functions for periodic or bounded solutions of ordinary differential equations, Discrete Contin. Dyn. Syst. 8 (2002) 39-54.

[40] V. Obukhovskii, P. Zecca, N.V. Loi, S. Kornev, Method of Guiding Functions in Problems of Nonlinear Analysis. Lecture Notes in Math. 2076, Springer-Velag, BerlinHeidelberg, 2013.

[41] B.J. Pettis, On the integration in vector spaces, Trans. Amer. Math. Soc. 44(1938) no. 2, 277-304.

[42] D. Rachinskii, Multivalent guiding functions in forced oscillation problems. Nonlinear Anal.: TMA. 26 (1996), no. 3, 631-639.

[43] L. Schwartz, Cours d'Analyse. 1. Second edition. Hermann, Paris, 1981.

[44] V. Taddei, Bound sets for Floquet boundary value problems: the nonsmooth case. Dis. Cont. Dyn. Sys., 6 (2000), no. 2, 459-473.

[45] F. Zanolin, Bound sets, periodic solutions and flow-invariant for ordinary differential equations in $\mathbb{R}^{n}$ : some remarks. Rend. Ist. Mat. Univ. Trieste 19(1987), 76-92. 